\title{
GEOMETRIC CONDITIONS FOR STRICT SUBMULTIPLICATIVITY OF RANK AND BORDER RANK
}

\author{
EDOARDO BALLICO, ALESSANDRA BERNARDI, FULVIO GESMUNDO, AND EMANUELE VENTURA \\ To Giorgio Ottaviani, on the occasion of his 60th birthday
}

\begin{abstract}
The $X$-rank of a point $p$ in projective space is the minimal number of points of an algebraic variety $X$ whose linear span contains $p$. This notion is naturally submultiplicative under tensor product. We study geometric conditions that guarantee strict submultiplicativity. We prove that in the case of points of rank two, strict submultiplicativity is entirely characterized in terms of the trisecant lines to the variety. Moreover, we focus on the case of curves: we prove that for curves embedded in an even-dimensional projective space, there are always points for which strict submultiplicativity occurs, with the only exception of rational normal curves.
\end{abstract}

\section{INTRODUCTION}

Let $V$ be a complex finite dimensional vector space and let $\mathbb{P} V$ be the corresponding projective space. When $V=\mathbb{C}^{n+1}$, denote $\mathbb{P} \mathbb{C}^{n+1}$ simply by $\mathbb{P}^{n}$. Throughout the paper, a projective, irreducible, reduced and linearly nondegenerate (i.e., not contained in a hyperplane) algebraic variety is called variety.

Ever since the nineteenth century, a line of research dealt with determining normal forms of algebraic objects in terms of basic building blocks: a classical example concerns expressions of homogeneous polynomials as sum of powers of linear forms. These additive problems can be rephrased equivalently in geometric terms as follows: given a variety and a point in its ambient space, determine sets of points of the variety whose linear span contains the given point. This approach motivated the study of secant varieties during the twentieth century. In the last decades the connections with applications involving additive tensor decomposition attracted the interest of a broad community, both in pure and applied mathematics and in other fields. In the rich literature, we briefly mention the classical [Cle61, Syl52, Pal03], concerning the study of homogeneous polynomials, [Pal06, Ad187], studying secant varieties of curves, [AR08, Str69, DVC00] drawing connection with phylogenetics, theoretical computer science and quantum information theory. We refer to [Lan12, $\left.\mathrm{BCC}^{+} 18\right]$ and the references therein for a more extensive presentation.

We formally introduce the notion of rank with respect to an arbitrary variety $X \subseteq \mathbb{P} V$. Given a point $p \in \mathbb{P} V$, the $X$-rank (or simply the rank) of $p$ is the minimal number of points of $X$ whose linear span contains $p$ :

$$
\mathrm{R}_{X}(p):=\min \left\{r \mid \exists q_{1}, \ldots, q_{s} \in X, p \in\left\langle q_{1}, \ldots, q_{s}\right\rangle\right\} .
$$

Let $\sigma_{r}(X)$ be the $r$-th secant variety of $X$, i.e., the Zariski-closure of the set of points whose $X$-rank is at most $r$. The border $X$-rank (or simply the border rank) of $p$ is

$$
\underline{\mathrm{R}}_{X}(p):=\min \left\{r \mid p \in \sigma_{r}(X)\right\} .
$$

2010 Mathematics Subject Classification. 15A69; 14N05, 14 H99.

Key words and phrases. rank, border rank, tensor product, Segre product, secant variety. 
It is natural to study properties of rank and border rank with respect to basic operations among varieties. Due to the additive nature of the problem, in the most general framework one is interested in relations between the rank of a linear combination of two points and their ranks. For example, in the tensor setting, Strassen [Str73] conjectured that the rank of a direct sum of two tensors always coincides with the sum of their ranks: this conjecture has been answered affirmatively in several cases [CCG12, BGL13, CCC15, Tei15, $\mathrm{CCC}^{+}$18, LM17, CCO17, BPR20]. In the case of tensors with three factors, a counterexample was given by Shitov [Shi19], while the analogous equality for border ranks was shown to be false already by Schönhage [Sch81]. In theoretical computer science, one is interested in multiplicativity properties of tensor rank and border rank under Kronecker powers, which capture the asymptotic complexity of the bilinear map defined by the tensor [Str87].

In this work, we are interested in multiplicativity properties of rank and border rank under tensor product. Given two varieties $X_{1} \subseteq \mathbb{P} V_{1}$ and $X_{2} \subseteq \mathbb{P} V_{2}$, their Segre product is the image of $X_{1} \times X_{2} \subseteq$ $\mathbb{P} V_{1} \times \mathbb{P} V_{2}$ under the Segre embedding

$$
\begin{array}{ccc}
\mathbb{P} V_{1} \times \mathbb{P} V_{2} & \longrightarrow \mathbb{P}\left(V_{1} \otimes V_{2}\right), \\
\left(\left[v_{1}\right],\left[v_{2}\right]\right) & \mapsto \quad\left[v_{1} \otimes v_{2}\right] .
\end{array}
$$

Denote the Segre product of $X_{1}$ and $X_{2}$ by $X_{1} \times X_{2}$. We often identify points in projective space and vectors of the line they represent; in particular, we will drop the bracket [-] from the notation.

For any $p_{1} \in \mathbb{P} V_{1}$ and $p_{2} \in \mathbb{P} V_{2}$, one has

$$
\mathrm{R}_{X_{1} \times X_{2}}\left(p_{1} \otimes p_{2}\right) \leq \mathrm{R}_{X_{1}}\left(p_{1}\right) \mathrm{R}_{X_{2}}\left(p_{2}\right) \quad \text { and } \quad \underline{\mathrm{R}}_{X_{1} \times X_{2}}\left(p_{1} \otimes p_{2}\right) \leq \underline{\mathrm{R}}_{X_{1}}\left(p_{1}\right) \underline{\mathrm{R}}_{X_{2}}\left(p_{2}\right) .
$$

Certain techniques to determine lower bounds on rank and border rank guarantee that the lower bound propagates to the tensor product and can be used to prove multiplicativity: this is the case of flattening lower bounds, see Section 4. However, both inequalities in (1) can be strict in general, as shown in [CJZ18] for rank and [CGJ19] for border rank.

Despite the achievements mentioned above originated from tensor problems, we investigate the multiplicativity problem in general.

Problem. Determine geometric conditions which guarantee either multiplicativity or strict submultiplicativity in the inequalities in (1).

\section{Contributions and structure of the paper.}

- In Section 2, we classify the ranks of tensor products of points of rank 2 : if $p_{1}, p_{2}$ are points of rank 2 with respect to varieties $X_{1}, X_{2}$ respectively, we give sufficient and necessary conditions so that the $\left(X_{1} \times X_{2}\right)$-rank of $p_{1} \otimes p_{2}$ is equal to 3 , instead of 4 ; see Theorem 2.6. As a corollary, if $p$ has $X$-rank 2, then $p^{\otimes 2}$ has $X^{\times 2}$-rank equal to 3 if and only if $p$ lies on a multisecant line.

- We show that if a variety $X$ admits a secant $r$-dimensional plane $\mathbb{P} W$ intersecting $X$ in more than $r+1$ points then, for any $p \in \mathbb{P} W$, the $X^{\times(r+1)}$-rank of $p^{\otimes(r+1)}$ is strictly less than $\mathrm{R}_{X \times(r+1)}(p)^{r+1}$; see Proposition 2.13 .

- In Section 3, we investigate conditions which guarantee that the geometric construction in [CGJ19] may be applied; see Proposition 3.4 and Proposition 3.8. We show that if $X \subseteq \mathbb{P}^{2 k}$ is a curve which is not the rational normal curve of degree $2 k$, then there are always examples of strict submultiplicativity; see Theorem 3.7.

- In Section 4, we turn our attention to homogeneous polynomials. In more geometric terms, we characterize rank submultiplicativity when either $X$ is a rational normal curve or $X$ is the third Veronese embedding of $\mathbb{P}^{2}$; see Proposition 4.1 and Proposition 4.2, respectively. 
- In Section 5, we consider cases for which multiplicativity of rank holds. We ask whether all minimal decompositions of products are products of minimal decompositions of the factors. In Theorem 5.4, we give conditions which guarantee a positive answer while in Example 5.6 we provide an example having a negative answer.

In view of these results, we propose the following.

Conjecture 1.1. Let $X \subseteq \mathbb{P}^{n}$ be a variety and let $p \in \mathbb{P}^{n}$.

$$
\text { If } \underline{\mathrm{R}}_{X}(p)<\mathrm{R}_{X}(p) \text {, then } \mathrm{R}_{X \times 2}(p \otimes p)<\mathrm{R}_{X}(p)^{2} \text {. }
$$

Observe that Conjecture 1.1 holds asymptotically, in the following sense. If $p \in \mathbb{P}^{n}$ is a point such that $\underline{\mathrm{R}}_{X}(p)<\mathrm{R}_{X}(p)$, then there is a value $k$ such that $\mathrm{R}_{X^{\times k}}\left(p^{\otimes k}\right)<\mathrm{R}_{X}(p)^{k}$ : this is a consequence of the fact that the two limits $\lim _{k \rightarrow \infty} \underline{\mathrm{R}}_{X^{\times k}}\left(p^{\otimes k}\right)^{1 / k}$ and $\lim _{k \rightarrow \infty} \mathrm{R}_{X^{\times k}}\left(p^{\otimes k}\right)^{1 / k}$ coincide and they are bounded from above by $\underline{\mathrm{R}}_{X}(p)$. We refer to [CGJ19, Section 6] for details on this asymptotic behaviour.

Acknowledgements. E.B. and A.B. acknowledge financial support from GNSAGA of INDAM (Italy). F.G. acknowledges financial support from the VILLUM FONDEN via the QMATH Centre of Excellence (Grant no. 10059). A.O. acknowledges financial support from the Alexander von Humboldt-Stiftung via a Humboldt Research Fellowship for Postdoctoral Researchers (April 2019 March 2021) at OVGU Magdeburg (Germany).

This collaboration started while F.G., A.O. and E.V. were visiting University of Trento for a Research in Pairs program at CIRM Trento in July 2018, continued while A.B. and F.G. were visiting the Institute for Computational and Experimental Research in Mathematics in Providence, RI, in Fall 2018, and was completed while A.B., F.G. and A.O. were visiting University of Pavia for the XXI Congresso dell'Unione Matematica Italiana, in September 2019. We thank CIRM, ICERM and UMI for providing good research environments to work on this project.

\section{Multisecant SPACES AND StRict submultiplicativity}

In this section, we look for geometric conditions on a variety $X$ which guarantee strict submultiplicaitivity for points of $X$-rank 2. The first idea comes from [CGJ19, Lemma 4.1] which shows that if the secant variety $\sigma_{r}(X)$ has a trisecant line $\mathbb{P} L$ such that one of the points of $\mathbb{P} L \cap \sigma_{r}(X)$ lies on $X$ itself, then there exists at least one point on $\mathbb{P} L$ for which the rank multiplicativity does not hold. We prove that strict rank submultiplicativity for points having $X$-rank 2 depends on the existence of a trisecant line to $X$ itself.

Definition 2.1. A line $\mathbb{P} L$ is multisecant to a variety $X \subset \mathbb{P} V$ if the intersection $X \cap \mathbb{P} L$ contains a set of at least 3 distinct points.

Notation 2.2. Given a vector space $V$, denote by $\operatorname{Sym}^{r} V$ the space of symmetric elements of $V^{\otimes r}$. Let $\nu_{r}$ be the map from $V$ to $\operatorname{Sym}^{r} V$ which sends $v \in V$ to $v^{\otimes r} \in \operatorname{Sym}^{r} V$. Denote by $\nu_{r}$ also the corresponding map between the projective spaces known as Veronese embedding. Given a subset $S \subseteq V$, denote by $\langle S\rangle$ the linear span of $S$ in $V$. Similarly, given a subset $S \subseteq \mathbb{P} V$, denote by $\langle S\rangle$ the projective linear span of $S$ in $\mathbb{P} V$.

Proposition 2.3. Let $X \subseteq \mathbb{P} V$ be a variety and let $\mathbb{P} L$ be a line such that $\mathbb{P} L \cap X$ contains a set of at least $k+1$ points. Let $p \in \mathbb{P} L \backslash X$. Then, for every $r \leq k$

$$
\mathrm{R}_{X \times r}\left(p^{\otimes r}\right) \leq r+1 .
$$

In particular, for every $r \geq 2, \mathrm{R}_{X \times r}\left(p^{\otimes r}\right)<\mathrm{R}_{X}(p)^{r}$. 
Proof. Notice that $\mathrm{R}_{X}(p)=2$, because $p$ lies on a secant line to $X$ and $p \notin X$. Let $L \subset V$ be the vector space of dimension 2 defining the projective line $\mathbb{P} L: p \in \mathbb{P} L$ and $p^{\otimes r}=\nu_{r}(p) \in \mathbb{P}\left(\operatorname{Sym}^{r} L\right)$. Fix $r \leq k$ and let $S \subseteq \mathbb{P} L \cap X$ be a set of $r+1$ points. Then, $\nu_{r}(S) \subseteq \nu_{r}(X) \subseteq X^{\times r}$ is a set of $r+1$ points lying on the rational normal curve $\nu_{r}(\mathbb{P} L) \subseteq \mathbb{P}\left(\operatorname{Sym}^{r} L\right) \subseteq \mathbb{P} V^{\otimes r}$. In particular, $\nu_{r}(S)$ is a set of $r+1$ linearly independent points in $\mathbb{P}\left(\operatorname{Sym}^{r} L\right) \simeq \mathbb{P}^{r}$; hence, $\left\langle\nu_{r}(S)\right\rangle=\mathbb{P}\left(\operatorname{Sym}^{r} L\right)$. Since $p^{\otimes r} \in\left\langle\nu_{r}(S)\right\rangle$, we conclude that $\mathrm{R}_{X^{\times r}}\left(p^{\otimes r}\right) \leq r+1$.

The inequality $\mathrm{R}_{X \times r}\left(p^{\otimes r}\right)<\mathrm{R}_{X}(p)^{r}$ for every $r \geq 2$ follows because if strict submultiplicativity holds for $r=2$, as shown above, then it holds for any $r \geq 2$.

Remark 2.4. More generally, the argument of Proposition 2.3 applies if $\mathbb{P} L \cap X$ is any 0-dimensional scheme rather than a set of points. The cactus $X$-rank of a point $p$ is the minimal degree of a 0 -dimensional scheme on $X$ whose linear span contains $p$ (see [RS11, BR13]). Then, similarly as for the $X$-rank, there is a notion of cactus varieties and border cactus $X$-rank (see [BR13, BB14]). Proposition 2.3 applies to cactus rank: if $\mathbb{P} L$ is a line whose intersection with $X$ is a 0 -dimensional scheme of degree at least $k+1$ then for every $r \leq k$, the cactus $X^{\times r}$-rank of $p^{\otimes r}$ is at most $r+1$.

Proposition 2.3 guarantees that for every point $p$ lying on a multisecant line of $X \subseteq \mathbb{P} V$, but not on $X$, multiplicativity of rank does not hold; in particular, the $X^{\times 2}$-rank of $p^{\otimes 2}$ is at most 3 . Theorem 2.6 below shows that this is essentially the only way that the tensor product of two elements of rank 2 has rank 3 instead of 4 .

First, we record an easy observation which will be used in the proof of Theorem 2.6.

Lemma 2.5. Let $p_{1} \otimes p_{2} \in \mathbb{P}\left(V_{1} \otimes V_{2}\right)$. Suppose $p_{1} \otimes p_{2} \in\left\langle a_{1} \otimes b_{1}, \ldots, a_{r} \otimes b_{r}\right\rangle$. Then, $p_{1} \in\left\langle a_{1}, \ldots, a_{r}\right\rangle$ and $p_{2} \in\left\langle b_{1}, \ldots, b_{r}\right\rangle$.

Proof. After passing to the underlying linear spaces, with a slight abuse of notation, we write $p_{1} \otimes p_{2}=\sum_{i} \lambda_{i} a_{i} \otimes b_{i} \in V_{1} \otimes V_{2}$. If $\beta \in V_{2}^{*}$ is a linear form such that $\beta\left(p_{2}\right) \neq 0$, then $\beta\left(p_{2}\right) p_{1}=$ $\sum_{i} \lambda_{i} \beta\left(b_{i}\right) a_{i}$ is a linear combination of $a_{1}, \ldots, a_{r}$ which gives $p_{1}$. Analogously, one can prove that $p_{2} \in\left\langle b_{1}, \ldots, b_{r}\right\rangle$.

Theorem 2.6. For $i=1,2$, let $X_{i} \subseteq \mathbb{P} V_{i}$ be varieties and let $p_{i} \in \mathbb{P} V_{i}$ such that $\mathrm{R}_{X_{i}}\left(p_{i}\right)=2$. Then, $3 \leq \mathrm{R}_{X_{1} \times X_{2}}\left(p_{1} \otimes p_{2}\right) \leq 4$. Moreover, for $a_{1}, a_{2}, a_{3} \in X_{1}$ and $b_{1}, b_{2}, b_{3} \in X_{2}$, the following are equivalent:

(i) $\mathrm{R}_{X_{1} \times X_{2}}\left(p_{1} \otimes p_{2}\right)=3$ with $p_{1} \otimes p_{2} \in\left\langle a_{1} \otimes b_{1}, a_{2} \otimes b_{2}, a_{3} \otimes b_{3}\right\rangle$;

(ii) the linear spaces $\mathbb{P} L_{1}=\left\langle a_{1}, a_{2}, a_{3}\right\rangle$ and $\mathbb{P} L_{2}=\left\langle b_{1}, b_{2}, b_{3}\right\rangle$ are multisecant lines to $X_{1}$ and $X_{2}$, respectively, where the $a_{i}$ 's and the $b_{i}$ 's are all distinct; moreover, if $\varphi: \mathbb{P} L_{1} \rightarrow \mathbb{P} L_{2}$ is the unique linear map such that $\varphi\left(a_{j}\right)=b_{j}$, then $\varphi\left(p_{1}\right)=p_{2}$.

Proof. The upper bound $\mathrm{R}_{X_{1} \times X_{2}}\left(p_{1} \otimes p_{2}\right) \leq 4$ is immediate from submultiplicativity.

First, we show the lower bound $3 \leq \mathrm{R}_{X_{1} \times X_{2}}\left(p_{1} \otimes p_{2}\right)$. Since $p_{1} \otimes p_{2} \notin X_{1} \times X_{2}$, we have $\mathrm{R}_{X_{1} \times X_{2}}\left(p_{1} \otimes p_{2}\right) \geq 2$. Suppose equality holds and $p_{1} \otimes p_{2} \in\left\langle a_{1} \otimes b_{1}, a_{2} \otimes b_{2}\right\rangle$, for $a_{1}, a_{2} \in X_{1}$ and $b_{1}, b_{2} \in X_{2}$. Let $\mathbb{P} L_{1}=\left\langle a_{1}, a_{2}\right\rangle$ and $\mathbb{P} L_{2}=\left\langle b_{1}, b_{2}\right\rangle$ : then $\operatorname{dim} \mathbb{P} L_{1}=\operatorname{dim} \mathbb{P} L_{2}=1$ otherwise $p_{1}=a_{1} \in X_{1}$ or $p_{2}=b_{1} \in X_{2}$. Now, regard $p_{1} \otimes p_{2}, a_{1} \otimes b_{1}$ and $a_{2} \otimes b_{2}$ as rank one matrices, after a suitable choice of coordinates, they can be identified with

$$
a_{1} \otimes b_{1}=\left(\begin{array}{cc}
1 & 0 \\
0 & 0
\end{array}\right) \quad \text { and } \quad a_{2} \otimes b_{2}=\left(\begin{array}{cc}
0 & 0 \\
0 & 1
\end{array}\right)
$$

then, $\left\langle a_{1} \otimes b_{1}, a_{2} \otimes b_{2}\right\rangle$ does not contain any other rank one matrix, providing a contradiction. This shows $\mathrm{R}_{X_{1} \times X_{2}}\left(p_{1} \otimes p_{2}\right) \geq 3$. 
Now, we address the second part of the statement. The same proof as in Proposition 2.3 (for $r=2$ ) shows that (ii) implies (i).

In order to prove that (i) implies (ii), we first show that $\operatorname{dim} \mathbb{P} L_{1}=\operatorname{dim} \mathbb{P} L_{2}=1$. Clearly $\operatorname{dim} \mathbb{P} L_{1}, \operatorname{dim} \mathbb{P} L_{2} \in\{1,2\}$, because if $\operatorname{dim} \mathbb{P} L_{i}=0, i=1,2$, then $p_{i} \in X_{i}$. Similarly to the first part of the proof, we reduce the problem to the span of rank one matrices:

- Let $\operatorname{dim} \mathbb{P} L_{1}=\operatorname{dim} \mathbb{P} L_{2}=2$. After a suitable choice of coordinates,

$$
a_{1} \otimes b_{1}=\left(\begin{array}{lll}
1 & 0 & 0 \\
0 & 0 & 0 \\
0 & 0 & 0
\end{array}\right), \quad a_{2} \otimes b_{2}=\left(\begin{array}{lll}
0 & 0 & 0 \\
0 & 1 & 0 \\
0 & 0 & 0
\end{array}\right), \quad a_{3} \otimes b_{3}=\left(\begin{array}{lll}
0 & 0 & 0 \\
0 & 0 & 0 \\
0 & 0 & 1
\end{array}\right),
$$

and their span does not contain other rank one matrices, providing a contradiction.

- Let $\operatorname{dim} \mathbb{P} L_{1}=2$ and $\operatorname{dim} \mathbb{P} L_{2}=1$. At least two among $b_{1}, b_{2}, b_{3}$ are distinct, so we may assume $b_{3} \in\left\langle b_{1}, b_{2}\right\rangle$. Passing to the affine cones, suppose $b_{3}=\lambda_{1} b_{1}+\lambda_{2} b_{2}$. After a suitable choice of coordinates,

$a_{1} \otimes b_{1}=\left(\begin{array}{cc}1 & 0 \\ 0 & 0 \\ 0 & 0\end{array}\right), \quad a_{2} \otimes b_{2}=\left(\begin{array}{ll}0 & 0 \\ 0 & 1 \\ 0 & 0\end{array}\right), \quad a_{3} \otimes b_{3}=a_{3} \otimes\left(\lambda_{1} b_{1}+\lambda_{2} b_{2}\right)=\left(\begin{array}{cc}0 & 0 \\ 0 & 0 \\ \lambda_{1} & \lambda_{2}\end{array}\right)$.

If $\lambda_{1}, \lambda_{2}$ are both nonzero, then their span does not contain other rank one matrices; contradiction. If $\lambda_{1}=0$, then $b_{3}=b_{2}$, and $p_{1} \otimes p_{2} \in\left\langle a_{1} \otimes b_{1}, a_{2} \otimes b_{2}, a_{3} \otimes b_{2}\right\rangle$, so that $p_{1} \otimes p_{2} \in\left\langle a_{1} \otimes b_{1}, \widetilde{a} \otimes b_{2}\right\rangle$, for some $\widetilde{a} \in\left\langle a_{2}, a_{3}\right\rangle$. Since $a_{1}, \widetilde{a}$ are linearly independent and $b_{1}, b_{2}$ are linearly independent, we obtain a contradiction as in the previous case; analogously for $\lambda_{2}=0$.

- Let $\operatorname{dim} \mathbb{P} L_{1}=1$ and $\operatorname{dim} \mathbb{P} L_{2}=2$. This is analogous to the previous case.

Therefore, we are left with the case $\operatorname{dim} \mathbb{P} L_{1}=\operatorname{dim} \mathbb{P} L_{2}=1$.

Claim. $\mathbb{P} L_{1}$ and $\mathbb{P} L_{2}$ are multisecant lines to $X_{1}$ and $X_{2}$, respectively.

Proof of Claim. We are going to show that $\sharp\left\{a_{1}, a_{2}, a_{3}\right\}=\sharp\left\{b_{1}, b_{2}, b_{3}\right\}=3$. Suppose, by contradiction, that the $a_{i}$ 's are not distinct, and assume $a_{2}=a_{3}$. There are two cases:

- if $b_{1}, b_{2}, b_{3}$ are distinct, then $\mathbb{P} L_{2}$ is a multisecant line to $X_{2}$ and we may assume $b_{3}=\lambda_{1} b_{1}+\lambda_{2} b_{2}$. In this case, after a suitable choice of coordinates,

$a_{1} \otimes b_{1}=\left(\begin{array}{cc}1 & 0 \\ 0 & 0\end{array}\right), \quad a_{2} \otimes b_{2}=\left(\begin{array}{cc}0 & 0 \\ 0 & 1\end{array}\right), \quad a_{3} \otimes b_{3}=a_{2} \otimes\left(\lambda_{1} b_{1}+\lambda_{2} b_{2}\right)=\left(\begin{array}{cc}0 & 0 \\ \lambda_{1} & \lambda_{2}\end{array}\right)$,

the only other rank one matrices in their span are of the form $\left(\begin{array}{ll}\mu_{1} & 0 \\ \mu_{2} & 0\end{array}\right)=\left(\mu_{1} a_{1}+\right.$ $\left.\mu_{2} a_{2}\right) \otimes b_{1}$ and this would imply $p_{2}=b_{1}$, in contradiction with the hypothesis;

- if $b_{1}, b_{2}, b_{3}$ are not distinct, then there are two possibilities: either $b_{1}=b_{2}$ (equivalently $b_{1}=b_{3}$ ) or $b_{2}=b_{3}$. Again, after a suitable choice of coordinates, we have one of the following possibilities:

- if $b_{1}=b_{2}$, then

$a_{1} \otimes b_{1}=\left(\begin{array}{ll}1 & 0 \\ 0 & 0\end{array}\right), \quad a_{2} \otimes b_{2}=a_{2} \otimes b_{1}=\left(\begin{array}{ll}0 & 0 \\ 1 & 0\end{array}\right), \quad a_{3} \otimes b_{3}=a_{2} \otimes b_{3}=\left(\begin{array}{ll}0 & 0 \\ 0 & 1\end{array}\right):$

the only other rank one matrices in their span are of the form $\left(\begin{array}{ll}\mu_{1} & 0 \\ \mu_{2} & 0\end{array}\right)=$ $\left(\mu_{1} a_{1}+\mu_{2} a_{2}\right) \otimes b_{1}$ or $\left(\begin{array}{cc}0 & 0 \\ \mu_{1} & \mu_{2}\end{array}\right)=a_{2} \otimes\left(\mu_{1} b_{1}+\mu_{2} b_{3}\right)$. In both cases we obtain a contradiction.

- if $b_{2}=b_{3}$, then $a_{2} \otimes b_{2}=a_{3} \otimes b_{3}$ and we would obtain $R_{X_{1} \times X_{2}}\left(p_{1} \otimes p_{2}\right)=2$, in contradiction with the first part of the proof. 
Therefore, we proved that $\mathbb{P} L_{1}$ (resp. $\mathbb{P} L_{2}$ ) is a multisecant line to $X_{1}$ (resp. $X_{2}$ ) and its intersection with $X$ contains the set of points $\left\{a_{1}, a_{2}, a_{3}\right\}$ (resp. the set of points $\left\{b_{1}, b_{2}, b_{3}\right\}$ ).

Let $\varphi: \mathbb{P} L_{1} \rightarrow \mathbb{P} L_{2}$ be the unique linear map such that $\varphi\left(a_{j}\right)=b_{j}$. Consider the identification $\varphi \times \operatorname{id}_{\mathbb{P} L_{2}}: \mathbb{P} L_{1} \times \mathbb{P} L_{2} \rightarrow \mathbb{P} L_{2} \times \mathbb{P} L_{2}$. By linearity, we have that $\varphi\left(p_{1}\right) \otimes p_{2} \in\left\langle\varphi\left(a_{1}\right) \otimes b_{1}, \varphi\left(a_{2}\right) \otimes\right.$ $\left.b_{2}, \varphi\left(a_{3}\right) \otimes b_{3}\right\rangle=\left\langle b_{1}^{\otimes 2}, b_{2}^{\otimes 2}, b_{3}^{\otimes 2}\right\rangle$. This shows that $\varphi\left(p_{1}\right) \otimes p_{2}$ is a symmetric rank one element of $\mathbb{P}\left(V_{2} \otimes V_{2}\right)$, namely it belongs to $\nu_{2}\left(\mathbb{P} L_{2}\right)$, so that $p_{2}=\varphi\left(p_{1}\right)$. This concludes the proof.

As a direct corollary, we have the following classification of the $X^{\times 2}$-ranks attained by $p^{\otimes 2}$ for points $p \in \mathbb{P} V$ with $X$-rank equal to 2: this is obtained by combining Theorem 2.6 and Proposition 2.3.

Corollary 2.7. Let $X \subset \mathbb{P} V$ be a variety. Let $p \in \mathbb{P} V$ with $\mathrm{R}_{X}(p)=2$. Then

$$
3 \leq \mathrm{R}_{X \times X}\left(p^{\otimes 2}\right) \leq 4,
$$

and $\mathrm{R}_{X \times X}\left(p^{\otimes 2}\right)=3$ if and only if $p$ lies on a multisecant line to $X$.

2.1. Examples of trisecant lines to space curves. The results we mention here are classical: we include them with a particular focus on characterizing points lying on generalized trisecant lines, namely those lines whose intersection with $X$ consists of a 0-dimensional scheme of degree at least 3 . A nondegenerate smooth curve $X \subset \mathbb{P}^{3}$ has infinitely many trisecant lines (their closure is a surface), unless the degree $d$ and genus $g$ of $X$ are $(d, g)=(3,0),(4,1)$; see [Ber98, Proposition 1, Remark 1]. When $X \subset \mathbb{P}^{3}$ is smooth (and in characteristic zero), the generic trisecant line has indeed three distinct points of intersection with the curve; this is a consequence of the results of [Kaj86].

Example 2.8 (Twisted cubic). If $(d, g)=(3,0)$, then $X$ is the twisted cubic, which has no trisecant line. Indeed, the ideal of the twisted cubic is generated by quadrics; hence, a trisecant line would have to be contained in every quadric surface containing $X$ and, in particular, in $X$. By Corollary 2.7, we recover a multiplicativity result for points with $X$-rank equal to 2 in the case of the twisted cubics: if $p \in \mathbb{P}^{3}$ satisfies $\mathrm{R}_{X}(p)=2$, then $\mathrm{R}_{X \times X}\left(p^{\otimes 2}\right)=4$.

Example 2.9 (Elliptic normal quartic). If $(d, g)=(4,1)$, then $X$ is an elliptic normal curve. The Riemann-Roch Theorem [Har77, Theorem IV.1.3] provides $h^{0}\left(\mathcal{O}_{X}(2)\right)=8$. To see this, let $K_{X}$ be the canonical divisor of $X$; recall $\operatorname{deg}\left(K_{X}\right)=2 g-2=0$. Thus the Riemann-Roch Theorem, applied to the divisor $D=2 H$ where $H$ is the hyperplane section, gives $h^{0}\left(\mathcal{O}_{X}(2)\right)=\operatorname{deg}\left(\mathcal{O}_{X}(2)\right)+g-1=8$. The long exact sequence in cohomology associated to the short exact sequence $0 \rightarrow \mathcal{I}_{X}(2) \rightarrow$ $\mathcal{O}_{\mathbb{P}^{3}}(2) \rightarrow \mathcal{O}_{X}(2) \rightarrow 0$ gives $h^{0}\left(\mathcal{I}_{X}(2)\right) \geq 2$. Thus $X$ is contained in the intersection of two quadric surfaces $Q_{1}, Q_{2}$. Since $\operatorname{deg}(X)=4=\operatorname{deg}\left(Q_{1} \cap Q_{2}\right), X$ is exactly the intersection of two quadrics. Every trisecant line to $X$ is contained in every quadric containing $X$, so $X$ has no trisecant lines. As above, if $p \in \mathbb{P}^{3}$ satisfies $\mathrm{R}_{X}(p)=2$, then $\mathrm{R}_{X \times X}\left(p^{\otimes 2}\right)=4$.

Example 2.10 (Rational quartic in $\left.\mathbb{P}^{3}\right)$. If $(d, g)=(4,0)$, then $X$ is a linear projection of the rational normal curve in $\mathbb{P}^{4}$. As before, by Riemann-Roch, $h^{0}\left(\mathcal{O}_{X}(2)\right)=9$. So the long exact sequence associated to $0 \rightarrow \mathcal{I}_{X}(2) \rightarrow \mathcal{O}_{\mathbb{P}^{3}}(2) \rightarrow \mathcal{O}_{X}(2) \rightarrow 0$ gives $h^{0}\left(\mathcal{I}_{X}(2)\right) \geq 1$.

Then $X$ is contained in at least one quadric surface $Q$. Recall that $Q$ is unique, irreducible and smooth:

- Uniqueness. If $Q$ is not unique, then $X$ would be a complete intersection of two quadrics and therefore would be of genus $g=1$.

- Irreducibility. If $Q$ is reducible, then it is union of two planes, in contradiction with the fact that $X$ is non-degenerate. 


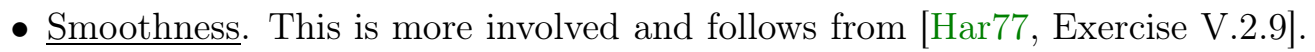

Notice that if $\mathbb{P} L$ is a trisecant line to $X$, then $\mathbb{P} L \subseteq Q$, therefore if $p \notin Q$ and $\mathrm{R}_{X}(p)=2$ then $p$ does not lie on a trisecant line and $\mathrm{R}_{X \times X}\left(p^{\otimes 2}\right)=4$.

We analyze points in $Q$. Since $Q$ is smooth, we have $Q \simeq \mathbb{P}^{1} \times \mathbb{P}^{1}$ and $X$ is a divisor on $Q$. Since $\operatorname{deg}(X)=4, X$ is a divisor of bidegree $(a, b)$ with $a+b=4$ : clearly $a, b>0$ because $X$ is non-degenerate. If $(a, b)=(2,2), X$ is the elliptic quartic of Example 2.9, in contradiction with the rationality of $X$. Therefore $X$ is a divisor of bidegree $(3,1)$ or $(1,3)$. Assume $X \in\left|\mathcal{O}_{Q}(3,1)\right|$. Now, lines in $Q$ coincide with the lines of the two rulings. If $\mathbb{P} L \in\left|\mathcal{O}_{Q}(0,1)\right|$, then $\operatorname{deg}(\mathbb{P} L \cap X)=$ $0 \cdot 3+1 \cdot 1=1$, so $\mathbb{P} L$ cannot be a trisecant line. If $\mathbb{P} L \in\left|\mathcal{O}_{Q}(1,0)\right|$, then $\operatorname{deg}(\mathbb{P} L \cap X)=1 \cdot 3+0 \cdot 1=3$, so that $\mathbb{P} L \cap X$ is a 0 -dimensional scheme of degree 3 , and $\mathbb{P} L$ is a trisecant line if and only if $\mathbb{P} L \cap X$ is reduced. Theorem 2.6 guarantees that the points lying on these lines are the unique elements of $X$-rank 2 for which strict submultiplicativity of rank occurs.

Let $\pi: X \rightarrow \mathbb{P}^{1}$ be the restriction of the projection of $Q \simeq \mathbb{P}^{1} \times \mathbb{P}^{1} \rightarrow \mathbb{P}^{1}$ on the first factor. Then $\pi$ is a finite morphism of degree 3 ; its fibers are generically reduced, showing that every line $\mathbb{P} L \in\left|\mathcal{O}_{Q}(1,0)\right|$ is a trisecant (with $L \cap X$ reduced) except at most finitely many of them. The lines in $\left|\mathcal{O}_{Q}(1,0)\right|$ for which $\mathbb{P} L \cap X$ is not reduced correspond to the ramification locus $\mathcal{R}$ of $\pi$. By Hurwitz Formula [Har77, Corollary 2.4], the degree of the ramification locus is $\operatorname{deg}(\mathcal{R})=2 g-2-2 d g+2 d=4$. This shows that there are at most 4 lines $\mathbb{P} L \in\left|\mathcal{O}_{Q}(1,0)\right|$ such that $\mathbb{P} L \cap X$ is not-reduced: equality holds if and only if the ramification locus is reduced; a more delicate argument, shows that indeed, there are always at least two distinct lines such that $\mathbb{P} L \cap X$ is not reduced.

Therefore, strict submultiplicaitivity of a point $p$ having $X$-rank 2 , occurs if and only if $p \in \mathbb{P} L \in$ $\left|\mathcal{O}_{Q}(1,0)\right|$ with $\mathbb{P} L \notin \mathcal{R}$.

Remark 2.11. For curves $X \subseteq \mathbb{P}^{4}$, one expects a finite number of trisecant lines. This number was determined by Castelnuovo and Berzolari, see [Bar06, p. 435]. If $X \subseteq \mathbb{P}^{4}$ has degree $d$ and genus $g$, then the expected number of trisecant lines is

$$
\frac{(d-2)(d-3)(d-4)}{6}-g(d-4)
$$

If $X \subseteq \mathbb{P}^{N}$ for $N \geq 5$ then one expects $X$ to have no trisecant lines, so Theorem 2.6 implies that the rank multiplicativity holds for points of $X$-rank 2 .

The idea of having a trisecant line to guarantee the strict rank multiplicativity of the $X$-rank 2 points can be extended to the existence of multisecant spaces of higher dimension.

\subsection{Multisecant $r$-dimensional planes.}

Definition 2.12. An $r$-dimensional linear space $\mathbb{P} W \simeq \mathbb{P}^{r}$ is multisecant to a variety $X \subset \mathbb{P} V$ if the intersection $\mathbb{P} W \cap X$ contains at least a set of $r+2$ distinct points.

Proposition 2.13. Let $X \subseteq \mathbb{P} V$ be a variety and $\mathbb{P} W \simeq \mathbb{P}^{r} \subseteq \mathbb{P} V$ be a multisecant linear space to $X$. Suppose that $\mathbb{P} W$ does not contain a multisecant $\mathbb{P}^{s}$, for any $s<r$. For every $p \in \mathbb{P} W$, we have

$$
\mathrm{R}_{X^{\times(r+1)}}\left(p^{\otimes(r+1)}\right) \leq(r+1)^{r+1}-(r+1) !+1 .
$$

Proof. Let $z_{0}, \ldots, z_{r}, w \in \mathbb{P} W \cap X$ so that $w \in\left\langle z_{0}, \ldots, z_{r}\right\rangle=\mathbb{P} W$. Since $\mathbb{P} W$ does not contain any smaller multisecant space, the point $w$ is not in the linear span of any proper subset of the $z_{i}$ 's. For every sequence of $(r+1)$ non-negative integers $\alpha=\left(\alpha_{0}, \ldots, \alpha_{r}\right)$ with $\alpha_{j} \leq r$, write $\mathbf{z}_{\alpha}=z_{\alpha_{0}} \otimes \cdots \otimes z_{\alpha_{r}}$. By definition,

$$
p^{\otimes(r+1)} \subseteq\left\langle\mathbf{z}_{\alpha}: \alpha \in\{0, \ldots, r\}^{r+1}\right\rangle=\mathbb{P} W^{\otimes(r+1)} \subseteq \mathbb{P} V^{\otimes(r+1)} .
$$


Let $\Sigma=\left\{\mathbf{z}_{\sigma} \mid \sigma \in \mathfrak{S}_{r+1}\right.$ is a permutation of $\left.\{0, \ldots, r\}\right\}$. Then, let

$$
\mathbb{P} E=\left\langle\mathbf{z}_{\alpha} \mid \mathbf{z}_{\alpha} \in \Sigma\right\rangle \quad \text { and } \quad \mathbb{P} F=\left\langle\mathbf{z}_{\alpha} \mid \mathbf{z}_{\alpha} \notin \Sigma\right\rangle .
$$

Clearly $\operatorname{dim} E=(r+1)$ !, $\operatorname{dim} F=(r+1)^{r+1}-(r+1)$ ! and both $E$ and $F$ are invariant under the action of $\mathfrak{S}_{r+1}$ which permutes the factors.

Claim. $p^{\otimes(r+1)} \in\left\langle\mathbb{P} F \cup w^{\otimes(r+1)}\right\rangle$.

Proof of Claim. If $p^{\otimes(r+1)} \in \mathbb{P} F$, then $p^{\otimes(r+1)} \in\left\langle\mathbb{P} F \cup w^{\otimes(r+1)}\right\rangle$.

Assume $p^{\otimes(r+1)} \notin \mathbb{P} F$. Consider the linear projection $\pi_{F}: \mathbb{P} W^{\otimes(r+1)} \rightarrow \mathbb{P} E$ from $\mathbb{P} F$, defined in terms of the chosen basis. Remark that $\pi_{F}$ is defined on $p^{\otimes(r+1)}$ since $p^{\otimes(r+1)} \notin \mathbb{P} F$. Moreover, $\pi_{F}$ is $\mathfrak{S}_{r+1}$-equivariant and, since $p^{\otimes(r+1)} \in \mathbb{P} W^{\otimes(r+1)}$ is symmetric, $\pi_{F}\left(p^{\otimes(r+1)}\right)$ is symmetric as well. The only symmetric element in $\mathbb{P} E$ is $e=\sum_{\sigma \in \mathfrak{S}_{r+1}} \mathbf{z}_{\sigma}$ and, therefore, we have that $\pi_{F}\left(p^{\otimes(r+1)}\right)=e$. Since $w$ is a non-trivial linear combination of all the $z_{i}$ 's, we deduce that $w^{\otimes(r+1)} \notin \mathbb{P} F$ and, in particular, $\pi_{F}$ is defined on $w^{\otimes(r+1)}$ so that $\pi_{F}\left(w^{\otimes(r+1)}\right)=e$ as well. Since $p^{\otimes(r+1)}$ and $w^{\otimes(r+1)}$ have the same image under $\pi_{F}$, the line $\left\langle w^{\otimes(r+1)}, p^{\otimes(r+1)}\right\rangle$ intersects $\mathbb{P} F$, showing that $p^{\otimes(r+1)} \in\left\langle\mathbb{P} F \cup w^{\otimes(r+1)}\right\rangle$.

Since $\mathbb{P} F$ is spanned by rank-one elements with respect to $X^{\times(r+1)}$, from the Claim we get that

$$
\mathrm{R}_{X^{\times(r+1)}}\left(p^{\otimes(r+1)}\right) \leq \operatorname{dim} F+1=(r+1)^{r+1}-(r+1) !+1 .
$$

\section{SUFFICIENT CONDITIONS FOR STRICT SUBMULTIPLICATIVITY OF BORDER RANK}

The existence of multisecant lines turns out to be a key tool for the strict submultiplicativity of the $X$-rank. The geometric concept of multisecant line can be generalized to what we will call $r$-multidrop lines; they turn out to be a valuable tool to verify the strict submultiplicativity of the border $X$-rank. In this section we study sufficient conditions that guarantee the existence of such $r$-multidrop lines that we introduce next.

3.1. The multidrop construction. The multidrop construction was firstly introduced in [CGJ19, Section 4]:

Definition 3.1. Let $X \subseteq \mathbb{P} V$ be a variety and let $z, q_{0}, q_{1}$ three points on a line $\mathbb{P} L \subseteq \mathbb{P} V$ such that

- $z \in \mathbb{P} L \cap X$;

- $q_{0}, q_{1} \in \mathbb{P} L \cap \sigma_{r}(X)$, with $q_{0}, q_{1}, z$ distinct;

- $\mathbb{P} L \nsubseteq \sigma_{r}(X)$.

The line $\mathbb{P} L$ is said to be an $r$-multidrop line for $X$.

Notice that in this case we have $\mathbb{P} L \subseteq \sigma_{r+1}(X)$. The existence of such a line guarantees the existence of points realizing strict submultiplicativity of border $X$-rank; for this reason, we call such a line an $r$-multidrop line for $X$. Note that 1-multidrop lines are simply multisecant lines in the sense of Definition 2.1.

Choose coordinates on $\mathbb{P} L \simeq \mathbb{P}^{1}$ such that $z=(1: 0), q_{0}=(0: 1)$ and $q_{1}=(1: 1)$. The line $\mathbb{P} L$ is parametrized by

$$
\ell: \begin{array}{ccc}
\mathbb{P}^{1} & \rightarrow & \mathbb{P} V \\
\left(\varepsilon_{0}: \varepsilon_{1}\right) & \mapsto & \ell\left(\varepsilon_{0}: \varepsilon_{1}\right)=\varepsilon_{0} z+\varepsilon_{1} q_{0},
\end{array}
$$


so that $\ell(1: 1)=q_{1}$. Let $p=q_{0}+2 z=q_{1}+z$. Points constructed in this way are used in [CGJ19] to obtain examples of strict submultiplicativity of border rank.

The following lemma was proved in [CGJ19] for a single variety $X$ and strict submultiplicativity for $p^{\otimes r}$ was considered. We state it more generally for the case of two distinct varieties; the proof is essentially the same.

Lemma 3.2 ([CGJ19, Lemma 4.1]). Let $X_{1} \subset \mathbb{P} V_{1}$ and let $X_{2} \subset \mathbb{P} V_{2}$. Let $\mathbb{P} L_{1}$ and $\mathbb{P} L_{2}$ such that $z_{i} \in \mathbb{P} L_{i} \cap X_{i}$ and $\left\{q_{i, 0}, q_{i, 1}\right\} \subseteq \mathbb{P} L_{i} \cap \sigma_{r_{i}}(X)$, for $i=1,2$, namely $\mathbb{P} L_{1}, \mathbb{P} L_{2}$ are $r_{1}$ - and $r_{2}$-multidrop lines for $X_{1}, X_{2}$, respectively. Let $p_{i}=q_{i, 0}+2 z_{i}$, for $i=1,2$. Then,

$$
\underline{\mathrm{R}}_{X_{1} \times X_{2}}\left(p_{1} \otimes p_{2}\right) \leq r_{1} r_{2}+r_{1}+r_{2}<\left(r_{1}+1\right)\left(r_{2}+1\right) .
$$

In particular, if $\underline{\mathrm{R}}_{X_{i}}\left(p_{i}\right)=r_{i}+1$, we obtain $\underline{\mathrm{R}}_{X_{1} \times X_{2}}\left(p_{1} \otimes p_{2}\right)<\underline{\mathrm{R}}_{X_{1}}\left(p_{1}\right) \cdot \underline{\mathrm{R}}_{X_{2}}\left(p_{2}\right)$.

The existence of multidrop lines is a strong condition and determining whether a variety admits them is not easy.

In [CGJ19], the case where $\sigma_{r}(X)$ is a hypersurface was considered. In this case, the existence of $r$-multidrop lines can be determined by studying the multiplicity of a point of $X$ in $\sigma_{r}(X)$. Briefly, suppose $\sigma_{r}(X)$ is a hypersurface, fix $z \in X$ and let $\mathbb{P} L$ be a generic line through $z$. By Bezout's Theorem, $\mathbb{P} L \cap \sigma_{r}(X)$ consists of $\operatorname{deg}\left(\sigma_{r}(X)\right)$ points counted with multiplicity and, by Bertini's Theorem, all intersection points except $z$ are non-singular points of $\sigma_{r}(X)$. More precisely, these are $\operatorname{deg}\left(\sigma_{r}(X)\right)-$ mult $_{\sigma_{r}(X)}(z)$ points, where $\operatorname{mult}_{\sigma_{r}(X)}(z)$ is the multiplicity of $z$ in $\sigma_{r}(X)$. In particular, if $\operatorname{deg}\left(\sigma_{r}(X)\right)-$ mult $_{\sigma_{r}(X)}(z) \geq 2$, then $L$ is a $r$-multidrop line and it is possible to apply Lemma 3.2.

In this section, we investigate conditions to guarantee that $\operatorname{deg}\left(\sigma_{r}(X)\right)-\operatorname{mult}_{\sigma_{r}(X)}(z) \geq 2$ in the hypersurface case.

First, notice that if $\operatorname{deg}\left(\sigma_{r}(X)\right)-$ mult $_{\sigma_{r}(X)}(z)=0$, then every line through $z$ only intersects $\sigma_{r}(X)$ at $z$; this implies that a line through $z$ and $q \in \sigma_{r}(X)$, for $q \in \sigma_{r}(X)$, must be entirely contained in $\sigma_{r}(X)$. This shows that $\sigma_{r}(X)$ is a cone with vertex containing $z$. In particular, we may assume that there exists $z \in X$ with $\operatorname{deg}\left(\sigma_{r}(X)\right)-$ mult $_{\sigma_{r}(X)}(z) \geq 1$, because if this is not the case, then $\sigma_{r}(X)$ is a cone over every point of $X$, against the non-degeneracy condition of $X$.

3.2. Rationality. First observe that if $\operatorname{deg}\left(\sigma_{r}(X)\right)-\operatorname{mult}_{\sigma_{r}(X)}(z)=1$, for some $z \in X$, then $\sigma_{r}(X)$ is a rational variety.

Proposition 3.3. Let $X \subseteq \mathbb{P}^{N}$ be a variety. Suppose that $\sigma_{r}(X)$ is a hypersurface and let $z \in X$ be a point with $\operatorname{deg}\left(\sigma_{r}(X)\right)-$ mult $_{\sigma_{r}(X)}(z)=1$. Then, $\sigma_{r}(X)$ is rational.

Proof. Let $\pi_{z}: \sigma_{r}(X) \rightarrow \mathbb{P}^{N-1}$ be the projection from $z$. The map $\pi_{z}$ is regular on $\sigma_{r}(X) \backslash\{z\}$. If $\mathbb{P} L$ is a generic line through $z$, then $\sigma_{r}(X) \cap \mathbb{P} L=\{z, q\}$, for a point $q \in \sigma_{r}(X)$. In particular, $q$ is the only point in $\pi_{z}^{-1}\left(\pi_{z}(q)\right)$, so $\pi_{z}$ is generically one-to-one and, therefore, birational.

Proposition 3.3 shows that if $\sigma_{r}(X)$ is not rational, then generic lines intersecting $X$ are multidrop lines. We provide a technical condition which guarantees that $\sigma_{r}(X)$ is not rational.

For a set $U$ and a positive integer $m$, let $\Sigma^{m} U:=U^{\times m} / \mathfrak{S}_{m}$ be the symmetric product of $m$ copies of $U$, that is the quotient of $U^{\times m}$ under the action of the symmetric group $\mathfrak{S}_{m}$ permuting the factors. If $X$ is a projective algebraic variety, then $\Sigma^{m} X$ is a projective algebraic variety, as well [Har92, Lecture 10]. The $r$-th abstract secant variety of $X \subseteq \mathbb{P}^{N}$ is

$$
\mathcal{S}_{r}^{\circ}(X):=\left\{\left(\left(x_{1}, \ldots, x_{r}\right), p\right) \in \Sigma^{r} X \times \mathbb{P}^{N}: p \in\left\langle x_{1}, \ldots, x_{r}\right\rangle\right\} \subseteq \Sigma^{r} X \times \mathbb{P}^{N} .
$$


Then $\mathcal{S}_{r}^{\circ}(X)$ is an irreducible locally closed set and the projection to $\mathbb{P}^{N}$ surjects onto the set of points having $X$-rank at most $r$. Write $\mathcal{S}_{r}(X)=\overline{\mathcal{S}_{r}^{\circ}(X)}$.

A variety $X$ is generically $r$-identifiable if the projection of $\mathcal{S}_{r}(X)$ to $\mathbb{P}^{N}$ is generically one-to-one, hence birational. In particular, if $X$ is generically $r$-identifiable, then $\sigma_{r}(X)$ is rational if and only if $\mathcal{S}_{r}(X)$ is rational. We refer to [BBC18] and the references therein for a complete explanation of the notion of identifiability and related topics.

Proposition 3.4. Let $X$ be generically $r$-identifiable and suppose there exists a non-constant map $\alpha: X \rightarrow A$ where $A$ is an abelian variety. Then, $\sigma_{r}(X)$ is not rational.

Proof. It is a known fact that if a variety $Y$ is rational and $A$ is an abelian variety, then every map $\beta: Y \rightarrow A$ is constant, see, e.g., [Mil08, Proposition 3.9]. Define $\alpha_{\Sigma}: \Sigma^{r} X \rightarrow A$ by $\alpha_{\Sigma}\left(x_{1}, \ldots, x_{r}\right)=$ $\alpha\left(x_{1}\right)+\cdots+\alpha\left(x_{r}\right)$, where + denotes the (abelian) operation of $A$ as a group; since + is commutative, $\alpha_{\Sigma}$ is well-defined. Notice that since $\alpha$ is not constant, then $\alpha_{\Sigma}$ is non-constant.

In particular, the map $\mathcal{S}_{r}(X) \rightarrow \Sigma^{r} X \rightarrow A$ given by the composition of the projection of $\mathcal{S}_{r}(X)$ onto $\Sigma^{r} X$ followed by $\alpha_{\Sigma}$ is non-constant as well. This shows that $\mathcal{S}_{r}(X)$ is not rational, and therefore, since by generic identifiability $\mathcal{S}_{r}(X)$ is birational to $\sigma_{r}(X)$, we conclude that $\sigma_{r}(X)$ is not rational.

We mention that, given a smooth projective variety $X$, there is a general construction to define an abelian variety $\operatorname{Alb}(X)$, called Albanese variety, together with a map $\alpha: X \rightarrow \operatorname{Alb}(X)$ satisfying a universal property, see [Bea96, Theorem V.13]. We do not provide any detail here, but we point out that one of the properties of this construction is that $\alpha(X)$ generates $\operatorname{Alb}(X)$ as an abelian group and that $\operatorname{dim} \operatorname{Alb}(X)=h^{1}\left(\mathcal{O}_{X}\right)$. In particular, if $h^{1}\left(\mathcal{O}_{X}\right)>0$, then $\alpha: X \rightarrow \operatorname{Alb}(X)$ is non-constant. Hence, Proposition 3.4 provides immediately the following.

Corollary 3.5. Let $X$ be a smooth projective variety, generically $r$-identifiable and such that $h^{1}\left(\mathcal{O}_{X}\right)>0$. Then, $\sigma_{r}(X)$ is not rational. Moreover, if $\sigma_{r}(X)$ is a hypersurface, then a generic line through $X$ is a r-multidrop line.

We conclude this section providing a class of varieties to which Corollary 3.5 can be applied.

Example 3.6. Let $X \subset \mathbb{P}^{2 k}$ be a smooth curve of genus $g>0$. By Palatini's Lemma (see, e.g., [Rus16, Corollary 1.2.3]), its $k$-th secant variety is a hypersurface. Generic identifiability always holds for curves, see [CC06, Corollary 2.7]. The condition $h^{1}\left(\mathcal{O}_{X}\right)>0$ follows by Serre's duality, since $h^{1}\left(\mathcal{O}_{X}\right)=h^{0}\left(\omega_{X}\right)=g$, where $\omega_{X}$ is the canonical bundle on $X$. This shows that $X$ satisfies the hypotheses of Corollary 3.5 and a generic line through $X$ is a $r$-multidrop line.

Example 3.6 generalizes the example of [CGJ19, Section 5.2] where it was shown that a generic line through the elliptic normal quintic $X \subseteq \mathbb{P}^{4}$ is a 2-multidrop. In that case, the result was shown directly using the equation of the hypersurface $\sigma_{2}(X)$ which provides $\operatorname{deg}\left(\sigma_{2}(X)\right)-\operatorname{mult}_{\sigma_{2}(X)}(z)=$ $5-3=2$. In fact, the result of the next subsection give a full generalization of Example 3.6.

3.3. The case of curves. We completely characterize the existence of multidrop lines for curves admitting a secant variety that is a hypersurface. Briefly, if $X \subseteq \mathbb{P}^{2 k}$ is a non-degenerate curve, then $X$ always admits $k$-multidrop lines, except if $X$ is the rational normal curve in $\mathbb{P}^{2 k}$.

Theorem 3.7. Let $X \subseteq \mathbb{P}^{2 k}$ be a non-degenerate curve and let $z \in X$ be a generic point. Then $\sigma_{k}(X)$ is a hypersurface. Moreover, $\operatorname{deg}\left(\sigma_{k}(X)\right)-\operatorname{mult}_{\sigma_{k}(X)}(z)=1$ if and only if $X$ is a rational normal curve of degree $2 k$. 
Proof. By Palatini's Lemma (see e.g. [Rus16, Proposition 1.2.3]), $\operatorname{dim} \sigma_{k}(X)=2 k-1$, so it is a hypersurface. Let $z \in X$ be a generic point and let $\mu=\operatorname{mult}_{\sigma_{k}(X)}(z)$. Let $\pi_{z}: \mathbb{P}^{2 k} \backslash\{z\} \rightarrow \mathbb{P}^{2 k-1}$ be the projection from $z$ and let $X^{\prime}=\overline{\pi_{z}(X)} \subseteq \mathbb{P}^{2 k-1}$. Since $z$ is generic, the projection $\pi_{z}$ is a birational map between $X$ and $X^{\prime}$; in particular $\pi_{z}$ is generically one-to-one on $X$. Moreover, $X^{\prime}$ is a non-degenerate curve and $\sigma_{k}\left(X^{\prime}\right)=\mathbb{P}^{2 k-1}$.

Let $\mathbb{P} L$ be a generic line through $z$. Let $q^{\prime}=\pi_{z}(\mathbb{P} L)$, which is a generic point of $\mathbb{P}^{2 k-1}$. Let $u: \mathcal{S}_{k}\left(X^{\prime}\right) \rightarrow \mathbb{P}^{2 k-1}$ be the projection from the abstract secant variety of $X^{\prime}$ to $\mathbb{P}^{2 k-1}$. Since $\operatorname{dim} \mathcal{S}_{k}\left(X^{\prime}\right)=2 k-1$, we deduce that $u$ is surjective and generically finite.

Since $q^{\prime}$ is generic in $\mathbb{P}^{2 k-1}, \operatorname{deg}(u)$ can be computed as the number of preimages of $q^{\prime}$ via $u$. Every element of $u^{-1}\left(q^{\prime}\right)$ is a pair $\left(S^{\prime}, q^{\prime}\right) \in \Sigma^{k} X^{\prime} \times \mathbb{P}^{2 k-1}$ where $S^{\prime} \subseteq X^{\prime}$ with $\left|S^{\prime}\right|=k$ and $q^{\prime} \in\left\langle S^{\prime}\right\rangle$. Now, from each element $\left(S^{\prime}, q^{\prime}\right) \in u^{-1}\left(q^{\prime}\right)$, we construct different points $q_{S^{\prime}} \in \sigma_{r}(X) \cap \mathbb{P} L$, such that all these points are also distinct from $z$. In particular, we conclude $\operatorname{deg}\left(\sigma_{k}(X)\right)-\mu \geq \operatorname{deg}(u)$.

Since $\pi_{z}$ is generically one-to-one on $X$ and $q^{\prime}$ is generic in $\mathbb{P}^{2 k-1}$, for every $\left(S^{\prime}, q^{\prime}\right) \in u^{-1}\left(q^{\prime}\right)$ and every $w^{\prime} \in S^{\prime}$, there is a unique $w \in X$ such that $\pi_{z}(w)=w^{\prime}$. In particular, every $\left(S^{\prime}, q^{\prime}\right) \in u^{-1}\left(q^{\prime}\right)$ defines a subset $S \subseteq X$ with $|S|=k$. Notice that for all $S$ constructed in this way, $z \notin\langle S\rangle^{\prime}$ : indeed, $\langle S\rangle$ is a generic $\mathbb{P}^{k-1}$ secant to $X$ and therefore by the Trisecant Lemma (see e.g. [Rus16, Proposition 1.4.3]), $\langle S\rangle$ only intersects $X$ at the points of $S$. For every $S$, let $q_{S^{\prime}}$ be the unique preimage of $q^{\prime}$ in $\langle S\rangle$. Notice that $q_{S^{\prime}}$ can only arise from a single $\left(S^{\prime}, q^{\prime}\right) \in u^{-1}\left(q^{\prime}\right)$. Indeed, $X$ is generically $r$-identifiable (see e.g. [CC02, Corollary 2.7]) and $q_{S^{\prime}}$ is a generic point of $\sigma_{k}(X)$, since it arises as intersection of $\sigma_{k}(X)$ with a generic line through $z$. In particular, for each $S^{\prime}$, the preimage $S$ is the only subset of $X$ with $|S|=k$ and $q_{S^{\prime}} \in\langle S\rangle$. This shows $\operatorname{deg}\left(\sigma_{k}(X)\right)-\mu \geq \operatorname{deg}(u)$.

Therefore, we conclude that $\operatorname{deg}\left(\sigma_{k}(X)\right)-\operatorname{mult}_{\sigma_{k}(X)}(z) \geq 2$ whenever $\operatorname{deg}(u) \geq 2$. By [CJ01, Theorem 3.4], the only curve $X^{\prime} \subseteq \mathbb{P}^{2 k-1}$ with $\operatorname{deg}(u)=1$ is a rational normal curve of degree $2 k-1$. If $X^{\prime}$ is a rational normal curve in $\mathbb{P}^{2 k-1}$ then $X$ is a rational normal curve in $\mathbb{P}^{2 k}$, showing that $\operatorname{deg}\left(\sigma_{k}(X)\right)-\operatorname{mult}_{\sigma_{k}(X)}(z) \geq 2$ if $X$ is not the rational normal curve.

Conversely, if $X$ is the rational normal curve in $\mathbb{P}^{2 k}$, then $\sigma_{k}(X)$ is the hypersurface of degree $k+1$ defined by the determinant of a square matrix of size $k+1$, see [Har92, Proposition 9.7]. In this case, one can observe mult $\sigma_{r}(X)(z)=k-1$ and therefore $\operatorname{deg}\left(\sigma_{k}(X)\right)-\operatorname{mult}_{\sigma_{k}(X)}(z) \geq 1$.

Theorem 3.7 generalizes Example 3.6 as well as the examples on curves of [CGJ19, Section 5.2 and Section 5.3].

3.4. Multidrop lines via projection. We saw that the existence of multidrop lines implies the existence of points for which border rank strict submultiplicativity occurs. The construction of the previous sections relies on the fact that one of the secant varieties is a hypersurface. In this section, we provide a more general construction to generate multidrop lines which does not depend on the dimension of the secant variety.

Let $X \subseteq \mathbb{P}^{N}$ and fix $r$ such that $\sigma_{r}(X) \neq \mathbb{P}^{N}$. Let $z \in X$ and $q_{0}, q_{1} \in \sigma_{r}(X)$ be generic points: in particular $z, q_{0}, q_{1}$ are not collinear and $\underline{\mathrm{R}}_{X}\left(q_{0}\right)=\mathrm{R}_{X}\left(q_{0}\right)=\underline{\mathrm{R}}_{X}\left(q_{1}\right)=\mathrm{R}_{X}\left(q_{1}\right)=r$. A generic $w \in\left\langle q_{0}, q_{1}, z\right\rangle$ is such that $\underline{\mathrm{R}}_{X}(w)=\mathrm{R}_{X}(w)=\min \left\{2 r+1, g_{X}\right\}$, where $g_{X}$ is the generic $X$-rank in $\mathbb{P}^{N}$.

Let $\pi_{w}: \mathbb{P}^{N} \rightarrow \mathbb{P}^{N-1}$ be the projection from $w$. Since $w \notin \sigma_{r}(X), \pi_{w}$ is regular on $\sigma_{r}(X)$ and by genericity $\underline{\mathrm{R}}_{\pi_{w}(X)}\left(\pi_{w}\left(q_{0}\right)\right)=\underline{\mathrm{R}}_{\pi_{w}(X)}\left(\pi_{w}\left(q_{1}\right)\right)=r$ and similarly for the $\pi_{w}(X)$-rank.

Proposition 3.8. In the construction above, let $\mathbb{P} L=\left\langle\pi_{w}(z), \pi_{w}\left(q_{0}\right)\right\rangle$. If $\mathbb{P} L \nsubseteq \sigma_{r}\left(\pi_{w}(X)\right)$, then $\mathbb{P} L$ is an $r$-multidrop line for $\pi_{w}(X)$. 
Proof. First observe that $\pi_{w}\left(q_{0}\right), \pi_{w}\left(q_{1}\right), \pi_{w}(z)$ are collinear, so that $\pi_{w}\left(q_{1}\right) \in \mathbb{P} L$ as well. Notice that $\pi_{w}\left(\sigma_{r}(X)\right) \subseteq \sigma_{r}\left(\pi_{w}(X)\right)$ because for every $x_{1}, \ldots, x_{r} \in X$, we have $w \notin\left\langle x_{1}, \ldots, x_{r}\right\rangle$, as $\mathrm{R}_{X}(w)>r$; in particular $\pi_{w}\left\langle x_{1}, \ldots, x_{r}\right\rangle=\left\langle\pi_{w}\left(x_{1}\right), \ldots, \pi_{w}\left(x_{r}\right)\right\rangle$. This shows that $\pi_{w}\left(q_{0}\right), \pi_{w}\left(q_{1}\right) \in$ $\sigma_{r}\left(\pi_{w}(X)\right)$.

By assumption $\mathbb{P} L \nsubseteq \sigma_{r}\left(\pi_{w}(X)\right)$, hence $\mathbb{P} L$ is a $r$-multidrop line for $\sigma_{r}\left(\pi_{w}(X)\right)$.

Proposition 3.8 generalizes the example of the rational quartic in $\mathbb{P}^{3}$ described in Section 2.10. Indeed, let $X$ be a smooth rational quartic in $\mathbb{P}^{3}$, then $X$ is realized as the projection of a rational normal quartic $Y \subseteq \mathbb{P}^{4}$ from a point $w \in \mathbb{P}^{4}$. Let $\pi_{w}: Y \rightarrow X$ be this projection.

If $\underline{\mathrm{R}}_{Y}(w) \leq 2$ then it is easy to verify that $\pi_{w}(X)$ is not smooth. Therefore, $\mathrm{R}_{Y}(w)=\underline{\mathrm{R}}_{Y}(w)=3$. Then, there are infinitely many planes $\left\langle y_{1}, y_{2}, y_{3}\right\rangle \subseteq \mathbb{P}^{4}$ with $y_{1}, y_{2}, y_{3} \in Y$ such that $w \in\left\langle y_{1}, y_{2}, y_{3}\right\rangle$. The images of $\pi_{w}\left(y_{1}\right), \pi_{w}\left(y_{2}\right), \pi_{w}\left(y_{3}\right)$ are collinear and the line $\mathbb{P} L=\left\langle\pi_{w}\left(y_{1}\right), \pi_{w}\left(y_{2}\right), \pi\left(y_{3}\right)\right\rangle$ is a trisecant line to $X=\pi_{w}(Y)$.

\section{Submultiplicativity for homogeneous polynomials}

This section studies submultiplicativity properties of rank of homogeneous polynomials with respect to Veronese varieties. Let $V$ be a vector space of dimension $n+1$ and let $\operatorname{Sym}^{d} V$ be the space of degree $d$ homogeneous polynomials in $n+1$ variables. The Veronese variety $X_{d}$ is defined as the image of the Veronese embedding

$$
\nu_{d}: \mathbb{P} V \longrightarrow \mathbb{P} \operatorname{Sym}^{d} V, \quad[\ell] \mapsto\left[\ell^{d}\right] .
$$

If $V$ is 2-dimensional, then $X_{d}$ is the rational normal curve $\mathcal{C}_{d} \subset \mathbb{P}^{d}$.

For homogeneous polynomials $f \in \operatorname{Sym}^{d} V$, the $X_{d}$-rank of the point $[f] \in \mathbb{P} \operatorname{Sym}^{d} V$ is classically referred to as Waring rank, and it coincides with the minimum number of $d$-th powers of linear forms needed to express $f$ as their linear combination. In the following, write $f$ both for the element in $\operatorname{Sym}^{d} V$ and for its projective class $[f]$.

The first example of rank strict submultiplicativity given in [CJZ18] was the monomial $x y^{2}$, for which $\mathrm{R}_{\mathcal{C}_{3}}\left(x y^{2}\right)=3$, but $\mathrm{R}_{\mathcal{C}_{3}^{\times 2}}\left(x y^{2} \otimes x y^{2}\right) \leq 8<9$. In fact, $\mathrm{R}_{\mathcal{C}_{3}^{\times 2}}\left(x y^{2} \otimes x y^{2}\right)=8$, see [CF18]. In [BBCG19], strict submultiplicativity was observed for all monomials of the form $x y^{d}$, for any $d \geq 3$. In this case, one has $\mathrm{R}_{\mathcal{C}_{d+1}}\left(x y^{d}\right)=d+1$ (see, e.g., [CCG12]), but $\mathrm{R}_{\mathcal{C}_{d+1}^{\times 2}}\left(x y^{d} \otimes x y^{d}\right) \leq 4 d+1<(d+1)^{2}$, see [BBCG19, Proposition 3.5]. Note that the form $x y^{d}$ lies on a tangent line to $\mathcal{C}_{d+1}$, so $\underline{\mathrm{R}}_{\mathcal{C}_{d}}\left(x y^{d}\right)=2$ and, therefore, it has border rank smaller than the rank. In particular, these are instances for which Conjecture 1.1 holds.

In this section, we study the cases of binary forms and ternary cubics, proving that Conjecture 1.1 holds in these cases. In fact, in these cases, having border rank strictly smaller than the rank is equivalent to have strict submultiplicativity for the rank of the second tensor power. However, we believe this to be a low dimensional phenomenon, due to the fact that in these cases the border rank lower bounds are attained by flattening methods, as explained below. We expect that as soon as there are cases of strict submultiplicativity of border rank, then strict submultiplicativity of rank will occur as well, even in examples where rank and border rank coincide. This is what happens in the tensor setting in the construction of [CGJ19] via the multidrop lines described in Section 3.

We introduce briefly some basics about flattening methods. A flattening of $\operatorname{Sym}^{d} V$ is a linear map Flat $: \operatorname{Sym}^{d} V \rightarrow \operatorname{Hom}(E, F)$ where $E, F$ are vector spaces. One can exploit semicontinuity of 
matrix rank to obtain lower bounds on $\underline{\mathrm{R}}(f)$ from the rank of Flat $(f)$ as a map from $E$ to $F$. More precisely, from [LO13, Proposition 4.1.1], we have

$$
\underline{\mathrm{R}}(f) \geq \frac{\operatorname{rank}(\operatorname{Flat}(f))}{\max \left\{\operatorname{rank}\left(\operatorname{Flat}\left(\ell^{d}\right)\right): \ell \in V\right\}} .
$$

Since Waring rank and Waring border rank are invariant under the action of the general linear group $G L(V)$ acting on the variables, usually one chooses Flat to be a $G L(V)$-equivariant map. In [CJZ18], it was shown that flattening lower bounds are multiplicative with respect to tensor products. This directly follows from: (a) the fact that one can combine two flattening maps by taking the Kronecker product of the images and (b) the rank of a linear map is multiplicative under Kronecker product. In [BBCG19, Section 7.2], generalizations to other varieties are given.

4.1. Binary forms. We recall basic facts of apolarity theory. We refer to [IK99, Ger96] for details.

Let $V$ be a vector space of dimension 2 and let $V^{*}$ be its dual. Write $\mathcal{C}_{d}=\nu_{d}(\mathbb{P} V)$ for the rational normal curve in $\mathbb{P} \operatorname{Sym}^{d} V$. Let $\{x, y\}$ be a basis for $V$. Then, we identify the symmetric algebra $\mathrm{Sym}^{\bullet} V$ with the polynomial algebra $\mathbb{C}[x, y]$ and $\mathrm{Sym}^{\bullet} V^{*}$ with the algebra of differential operators with constant coefficients $\mathbb{C}\left[\partial_{x}, \partial_{y}\right]$, where $\partial_{x}=\frac{\partial}{\partial x}$ and $\partial_{y}=\frac{\partial}{\partial y}$. Then, Sym $V^{*}$ acts on $\operatorname{Sym} \bullet V$ by contraction with the differential operators:

$$
\circ: \operatorname{Sym}^{\bullet} V^{*} \times \operatorname{Sym}^{\bullet} V \longrightarrow \operatorname{Sym}^{\bullet} V, \quad(\varphi, f) \mapsto \varphi \circ f:=\varphi(f) .
$$

The apolar ideal of a homogeneous polynomial $f \in \operatorname{Sym}^{d} V$, denoted $f^{\perp} \subset \operatorname{Sym} \bullet V^{*}$, is the ideal of differential operators which annihilate $f$, i.e., $f^{\perp}=\left\{\varphi \in \operatorname{Sym}^{\bullet} V^{*} \mid \varphi \circ f=0\right\}$. It is a classical result that if $f$ is a binary form, then

$$
f^{\perp}=\left(\varphi_{1}, \varphi_{2}\right), \quad \text { where } \operatorname{deg}\left(\varphi_{1}\right)+\operatorname{deg}\left(\varphi_{2}\right)=\operatorname{deg}(f)+2 .
$$

If $\operatorname{deg}\left(\varphi_{1}\right) \leq \operatorname{deg}\left(\varphi_{2}\right)$ then $\underline{\mathrm{R}}_{\mathcal{C}_{d}}(f)=\operatorname{deg}\left(\varphi_{1}\right)$. Moreover, if $\varphi_{1}$ is square-free (as a homogeneous polynomial in $\left.\partial_{x}, \partial_{y}\right)$, then $\mathrm{R}_{\mathcal{C}_{d}}(f)=\operatorname{deg}\left(\varphi_{1}\right)$, otherwise, $\mathrm{R}_{\mathcal{C}_{d}}(f)=\operatorname{deg}\left(\varphi_{2}\right)$. As a consequence, the rank of a generic binary form of degree $d$ is $\left\lceil\frac{d+1}{2}\right\rceil$. These results essentially date back to Sylvester [Sy151]; for more recent references, see [IK99, CS11, BGI11].

The following result proves a stronger version of Conjecture 1.1 in the case of binary forms.

Theorem 4.1. Let $f \in \operatorname{Sym}^{d} V, \operatorname{dim} V=2$. Then,

$$
\underline{\mathrm{R}}_{\mathcal{C}_{d}}(f)<\mathrm{R}_{\mathcal{C}_{d}}(f) \quad \text { if and only if } \quad \mathrm{R}_{\mathcal{C}_{d}^{\times 2}}\left(f^{\otimes 2}\right)<\mathrm{R}_{\mathcal{C}_{d}}(f)^{2} .
$$

Proof. First, we show that if $\underline{\mathrm{R}}_{\mathcal{C}_{d}}(f)=\mathrm{R}_{\mathcal{C}_{d}}(f)$ then multiplicativity holds. This is a consequence of multiplicativity of flattening lower bounds. Indeed, for every $e \leq d$, define the following flattening map, called $e$-th catalecticant:

$$
\begin{array}{ccc}
\operatorname{cat}_{e}: \operatorname{Sym}^{d} V & \longrightarrow \operatorname{Hom}\left(\operatorname{Sym}^{e} V^{*}, \operatorname{Sym}^{d-e} V\right) \\
f & \mapsto \operatorname{cat}_{e}(f): \varphi \mapsto \varphi \circ f .
\end{array}
$$

Equivariantly, cat ${ }_{e}$ is an embedding of $\operatorname{Sym}^{d} V$ into $\operatorname{Sym}^{e} V \otimes \operatorname{Sym}^{d-e} V$.

It is easy to see that if $f$ is a binary form with $\underline{\mathrm{R}}(f)=r$, then $\operatorname{rank}\left(\operatorname{cat}_{e}(f)\right) \leq r$ with equality for $r-1 \leq e \leq d-(r-1)$ (this is a classical result that goes back to Sylvester [Syl51]). In particular, the denominator in (2) is one and the border rank of binary forms matches a flattening lower bound.

By multiplicativity of flattening lower bounds, we deduce $\mathrm{R}_{\mathcal{C}_{d}^{\times 2}}(f \otimes f)=\underline{\mathrm{R}}_{\mathcal{C}_{d}^{\times 2}}(f \otimes f)=r^{2}$.

Now, suppose that $\underline{\mathrm{R}}_{\mathcal{C}_{d}}(f)<\mathrm{R}_{\mathcal{C}_{d}}(f)$. From the discussion above, $f^{\perp}=\left(\varphi_{1}, \varphi_{2}\right)$ with $\underline{\mathrm{R}}_{\mathcal{C}_{d}}(f)=$ $\operatorname{deg}\left(\varphi_{1}\right)<\operatorname{deg}\left(\varphi_{2}\right)=\mathrm{R}_{\mathcal{C}_{d}}(f)$ and $\mathrm{R}_{\mathcal{C}_{d}}(f)+\underline{\mathrm{R}}_{\mathcal{C}_{d}}(f)=d+2$. In particular, if $r_{g}=\left\lceil\frac{d+1}{2}\right\rceil$ is the generic rank in $\mathbb{P} \operatorname{Sym}^{d} V$, we have $\mathrm{R}_{\mathcal{C}_{d}}(f) \geq r_{g}+1$. Write $\mathrm{R}_{\mathcal{C}_{d}}(f)=\left(r_{g}+1\right)+\rho$ for some $\rho \geq 0$. 
We use induction on $\rho$.

Case $\rho=0$, i.e., $\mathrm{R}_{\mathcal{C}_{d}}(f)=r=r_{g}+1$. For any $s$, let $Y_{s}$ be the locus of forms having rank exactly $s$.

Claim. $Y_{r_{g}}$ is Zariski-open.

Proof of Claim. We use [CS11, Theorem 11]. Write $d=2 \delta$ or $d=2 \delta+1$ depending on the parity; then $r_{g}=\delta+1$. If $d=2 \delta$ is even, $Y_{r_{g}}$ is the complement of $\sigma_{\delta}\left(\mathcal{C}_{d}\right)$, hence Zariski-open. If $d=2 \delta+1$ is odd, then $Y_{r_{g}}$ is the complement of the union $\sigma_{\delta}\left(\mathcal{C}_{d}\right) \cup Y_{\delta+2}$. Write $Y_{r_{g}}=\mathbb{P} \operatorname{Sym}^{d} V \backslash\left(\sigma_{\delta}\left(\mathcal{C}_{d}\right) \cup Y_{\delta+2}\right)=\left(\mathbb{P} \operatorname{Sym}^{d} V \backslash \sigma_{\delta}\left(\mathcal{C}_{d}\right)\right) \backslash Y_{\delta+2}$. Since $\sigma_{\delta}\left(\mathcal{C}_{d}\right)$ is closed, $\mathbb{P} \operatorname{Sym}^{d} V \backslash \sigma_{\delta}\left(\mathcal{C}_{d}\right)$ is open and it suffices to show that $Y_{\delta+2}$ is a closed subset of $\mathbb{P} \operatorname{Sym}^{d} V \backslash \sigma_{\delta}\left(\mathcal{C}_{d}\right)$. This can be shown as follows. Let $h \in Y_{\delta+2}$, namely $\mathrm{R}_{\mathcal{C}_{d}}(h)=\delta+2$ so that $\underline{\mathrm{R}}_{\mathcal{C}_{d}}(h)=\delta+1$; the apolar ideal of $h$ is $h^{\perp}=\left(\psi_{1}, \psi_{2}\right)$ with $\operatorname{deg}\left(\psi_{1}\right)=\delta+1$ and $\psi_{1}$ not square-free. Therefore, $Y_{\delta+2}$ is determined by the vanishing of the discriminant of $\psi_{1}$; indeed, the generator of lowest degree in the apolar ideal of an element of $Y_{\delta+1}$ has degree $\delta+1$ and it is square-free; since the vanishing of the discriminant is a closed condition, we conclude.

Now, let $\ell$ be a linear form such that there exists a scalar $a$ with $\mathrm{R}_{\mathcal{C}_{d}}\left(f-a \ell^{d}\right)=\mathrm{R}_{\mathcal{C}_{d}}(f)-1$, namely $f-a \ell^{d} \in Y_{r_{g}}$. Since $Y_{r_{g}}$ is Zariski-open by the Claim, we deduce that $f-\varepsilon \ell^{d} \in Y_{r_{g}}$ for a generic choice of $\varepsilon$. In particular, let $\varepsilon$ be such that $q_{0}=f-2 \varepsilon \ell^{d}$ and $q_{1}=f-\varepsilon \ell^{d}$ are elements of $Y_{r_{g}}$. We conclude using the same argument as in [CJZ18]: we write

$$
f \otimes f=\left(q_{0}+2 \varepsilon \ell^{d}\right) \otimes\left(q_{0}+2 \varepsilon \ell^{d}\right)=q_{0} \otimes q_{0}+q_{1} \otimes \varepsilon \ell^{d}+\varepsilon \ell^{d} \otimes q_{1}
$$

and we deduce $\mathrm{R}_{\mathcal{C}_{d}^{\times 2}}(f \otimes f) \leq(r-1)^{2}+2(r-1)=r^{2}-1<\mathrm{R}_{\mathcal{C}_{d}}(f)^{2}$.

Case $\rho \geq 1$. Let $\ell$ be a linear form such that $\mathrm{R}_{\mathcal{C}_{d}}\left(f-\ell^{d}\right)=\mathrm{R}(f)-1$. Let $g=f-\ell^{d}$ and observe that $\mathrm{R}_{\mathcal{C}_{d}}(g)=r-1=\left(r_{g}+1\right)+\rho-1 \geq r_{g}+1$. In particular $\mathrm{R}_{\mathcal{C}_{d}^{\times 2}}(g \otimes g) \leq \mathrm{R}_{\mathcal{C}_{d}}(g)^{2}-1=(r-1)^{2}-1$, by the induction hypothesis. Hence, we write

$$
f \otimes f=\left(g-\ell^{d}\right) \otimes\left(g-\ell^{d}\right)=g \otimes g-g \otimes \ell^{d}-\ell^{d} \otimes g+\ell^{d} \otimes \ell^{d},
$$

and, passing to the rank, we obtain

$$
\mathrm{R}_{\mathcal{C}_{d}^{\times 2}}(f \otimes f) \leq\left[(r-1)^{2}-1\right]+(r-1)+(r-1)+1=r^{2}-1 .
$$

4.2. Plane cubics. Also in the case of ternary cubics we prove a stronger version of Conjecture 1.1. Let $X_{3}$ be the Veronese surface given by the Veronese embedding of $\mathbb{P} V$ in $\mathbb{P} \operatorname{Sym}^{3} V$, with $\operatorname{dim} V=3$.

There is a complete classification of plane cubics, up to change of coordinates: in other words, the $G L(V)$-orbits in $\mathrm{Sym}^{3} V$ are entirely classified, together with ranks and border ranks of their elements, see e.g. [KM02, LT10]. We record them as in [CCO17] for convenience:

(i) $f=x^{3}+y^{3}+z^{3}+a \cdot x y z$ with $a^{3} \neq-3^{3}, 0,6^{3}$ : the generic cubic, with $\underline{\mathrm{R}}(f)=\mathrm{R}(f)=4$;

(ii) $f=x^{3}+y^{3}+z^{3}$ : the Fermat cubic, with $\underline{\mathrm{R}}(f)=\mathrm{R}(f)=3$;

(iii) $f=x^{2}(x-z)+y^{2} z$ : the nodal cubic with $\underline{\mathrm{R}}(f)=\mathrm{R}(f)=4$;

(iv) $f=x^{3}+y^{2} z$ : the cuspidal cubic with $\underline{\mathrm{R}}(f)=3, \mathrm{R}(f)=4$;

(v) $f=z\left(x^{2}+y^{2}+z^{2}\right)$ : a conic and a secant line, with $\underline{\mathrm{R}}(f)=\mathrm{R}(f)=4$;

(vi) $f=z\left(x^{2}+y z\right)$ : a conic and a tangent line, with $\underline{\mathrm{R}}(f)=3, \mathrm{R}(f)=5$;

(vii) $f=x y z$ : three lines intersecting generically, with $\underline{\mathrm{R}}(f)=\mathrm{R}(f)=4$; 
(viii) $f=x y(x+y)$ : three lines intersecting in a single point, with $\underline{\mathrm{R}}(f)=\mathrm{R}(f)=2$;

(ix) $f=x^{2} y$ : a double line and a transverse line, with $\underline{\mathrm{R}}(f)=2, \mathrm{R}(f)=3$;

(x) $f=x^{3}$ : a triple line, with $\underline{\mathrm{R}}(f)=\mathrm{R}(f)=1$.

Proposition 4.2. Let $f \in \operatorname{Sym}^{3} V$, $\operatorname{dim} V=3$. Then,

$$
\mathrm{R}_{X_{3}^{\times 2}}(f \otimes f)<\mathrm{R}_{X_{3}}(f)^{2} \quad \text { if and only if } \quad \mathrm{R}_{X_{3}}(f)<\underline{\mathrm{R}}_{X_{3}}(f) .
$$

Proof. The cases (viii), (ix) and (x) are already included in Theorem 4.1. Hence, it suffices to show that $\mathrm{R}_{X_{3}^{\times 2}}(f \otimes f)=\mathrm{R}_{X_{3}}(f)^{2}$ in cases (i), (ii), (iii), (v), (vii) and $\mathrm{R}_{X_{3}^{\times 2}}(f \otimes f)<\mathrm{R}_{X_{3}}(f)^{2}$ in cases (iv), (vi).

The equality in cases (i), (ii), (iii), (v), (vii) follows by multiplicativity of flattening lower bounds, which implies multiplicativity of border rank. For $f \in \operatorname{Sym}^{3} V$, define the linear map $f^{\wedge 1}$ given by the following composition

$$
f^{\wedge 1}: V \otimes V^{*} \stackrel{\operatorname{id}_{V} \otimes \operatorname{cat}_{1}(f)}{\longrightarrow} V \otimes \operatorname{Sym}^{2} V \stackrel{\delta}{\longrightarrow} \Lambda^{2} V \otimes V,
$$

where the first map is the catalecticant map augmented via the identity on a factor $V$ and the second map $\delta: V \otimes \operatorname{Sym}^{2} V \rightarrow \Lambda^{2} V \otimes V$ is the classical Koszul differential [Eis95, Chapter 17]. It is a classical fact that $\underline{\mathrm{R}}_{X_{3}}(f)=4$ if and only if $\operatorname{rank}\left(f^{\wedge 1}\right)=8$; see [Lan12, Section 3.10]. In particular, the flattening map $\operatorname{Sym}^{3} V \rightarrow \operatorname{Hom}\left(V \otimes V^{*}, \Lambda^{2} V \otimes V\right)$ provides a flattening lower bound in the cases (i), (ii), (iii), (v), (vii), which is multiplicative.

Strict submultiplicativity in the case (iv) follows directly from the submultiplicativity for the monomial $y^{2} z$. Indeed, we have

$$
\begin{aligned}
\mathrm{R}_{X_{3}^{\times 2}}\left(\left(x^{3}+y^{2} z\right) \otimes\right. & \left.\left(x^{3}+y^{2} z\right)\right) \\
& \leq \mathrm{R}_{X_{3}^{\times 2}}\left(x^{3} \otimes x^{3}\right)+\mathrm{R}_{X_{3}^{\times 2}}\left(y^{2} z \otimes x^{3}\right)+\mathrm{R}_{X_{3}^{\times 2}}\left(x^{3} \otimes y^{2} z\right)+\mathrm{R}_{X_{3}^{\times 2}}\left(y^{2} z \otimes y^{2} z\right) \\
& =1+3+3+8=15<16=\left(\mathrm{R}_{X_{3}}\left(x^{3}+y^{2} z\right)\right)^{2} .
\end{aligned}
$$

Consider the case (vi), i.e., $f=z\left(x^{2}+y z\right)$. Let, $f_{\varepsilon}=f-\varepsilon y^{3}$. Then, $\operatorname{rank}\left(f_{1}^{\wedge 1}\right)=8$, showing $\underline{\mathrm{R}}_{X_{3}}\left(f_{\varepsilon}\right)=4$, for a generic choice of $\varepsilon$. From the classification, observe that $\mathrm{R}_{X_{3}}\left(g_{\varepsilon}\right)=4$ as well. In particular, there exists a coefficient $\varepsilon$ such that both $f_{\varepsilon}$ and $f_{2 \varepsilon}$ have rank 4. Proceeding as in (3), we conclude $\mathrm{R}_{X_{3}}\left(f^{\otimes 2}\right) \leq 24$.

4.3. A lower bound for rank of tensor product of bivariate monomials. We conclude this section providing a lower bound on the rank of the tensor product of two monomials in two variables. We use a method introduced in [CCG12].

Let $m=x^{a} y^{b}$, with $a \leq b$. It is known that $\mathrm{R}_{\mathcal{C}_{a+b}}(m)=b+1$ (see e.g. [BGI11, Remark 24], [CCG12, Proposition 3.1] and [RS11, Corollary 2]) and that $\underline{\mathrm{R}}_{\mathcal{C}_{a+b}}(m)=a+1$ (see e.g. [LT10, Theorem 1.11] and [RS11, Corollary 2]). By Theorem 4.1, we have that $\mathbf{R}_{\mathcal{C}_{a+b}^{\times 2}}\left(m^{\otimes 2}\right)=\mathbf{R}_{\mathcal{C}_{a+b}}(m)^{2}$ if and only if $a=b$. From [BBCG19, Proposition 4.3], we have the lower bound $\mathrm{R}_{\mathcal{C}_{d}^{\times 2}}(m \otimes m) \geq 2 d-1$ when $m=x y^{d-1}$. We provide a lower bound for any $x^{a} y^{b}$ with $a<b$.

The strategy of the proof allows us to formulate the result for the tensor product of two binary monomials, not necessarily equal.

Proposition 4.3. Let $m_{1}=x_{1}^{a_{1}} y_{1}^{b_{1}}$ and $m_{2}=x_{2}^{a_{2}} y_{2}^{b_{2}}$ with $a_{1} \leq b_{1}$ and $a_{2} \leq b_{2}$. Then,

$$
\mathrm{R}_{\mathcal{C}_{a_{1}+b_{1}} \times \mathcal{C}_{a_{2}+b_{2}}}\left(m_{1} \otimes m_{2}\right) \geq \max \left\{\left(a_{1}+1\right)\left(b_{2}+1\right),\left(a_{2}+1\right)\left(b_{1}+1\right)\right\} .
$$


Proof. We show $\mathrm{R}_{\mathcal{C}_{a_{1}+b_{1}} \times \mathcal{C}_{a_{2}+b_{2}}}\left(m_{1} \otimes m_{2}\right) \geq\left(a_{1}+1\right)\left(b_{2}+1\right)$.

Regard $m_{1} \otimes m_{2}$ in the bi-graded polynomial ring $S=\mathbb{k}\left[x_{1}, y_{1}\right] \otimes \mathbb{k}\left[x_{2}, y_{2}\right]=\mathbb{k}\left[x_{1}, y_{1} ; x_{2}, y_{2}\right]$, where $\operatorname{deg}\left(x_{1}\right)=\operatorname{deg}\left(y_{1}\right)=(1,0)$ and $\operatorname{deg}\left(x_{2}\right)=\operatorname{deg}\left(y_{2}\right)=(0,1)$. Let $S_{(i, j)}$ be the $\mathbb{k}$-vector space of homogeneous polynomials of bi-degree $(i, j)$. The apolar ideal of $m_{1} \otimes m_{2}$ is

$$
\left(m_{1} \otimes m_{2}\right)^{\perp}=\left(x_{1}^{a_{1}+1}, y_{1}^{b_{1}+1}, x_{2}^{a_{2}+1}, y_{2}^{b_{2}+1}\right) .
$$

From the multigraded version of the Apolarity Lemma (see e.g. [GRV16, Gał16, BBCM11, BBG19, Ber08]), the rank of $m_{1} \otimes m_{2}$ coincides with the minimal cardinality of a reduced set of points $\mathbb{X} \subset \mathbb{P}^{1} \times \mathbb{P}^{1}$ whose bi-graded defining ideal $I_{\mathbb{X}}$ is contained in $\left(m_{1} \otimes m_{2}\right)^{\perp}$. For any bi-graded ideal $I \subset S$, we denote $I_{(i, j)}=I \cap S_{(i, j)}$. The Hilbert function of $S / I$ in degree $(i, j)$ is $\operatorname{HF}_{S / I}(i, j):=$ $\operatorname{dim}_{\mathbb{k}} S_{(i, j)} / I_{(i, j)}$. We refer to [SVT06] for basic properties of the bi-graded Hilbert function of ideals of points in multi-projective space. For convenience, we just recall two facts about the Hilbert function of ideals of points that we will use in the rest of the proof:

(1) the Hilbert function of $S / I_{\mathbb{X}}$ is strictly increasing until it gets constant along the $i$-th row $\left(\operatorname{HF}_{S / I_{\mathbb{X}}}(i, j)\right)_{j \geq 0}$ and along the $j$-th column $\left(\operatorname{HF}_{S / I_{\mathbb{X}}}(i, j)\right)_{i \geq 0}$;

(2) if $i, j \gg 0$, then $\operatorname{HF}_{S / I_{\mathbb{X}}}(i, j)=|\mathbb{X}|$.

Let $\mathbb{X} \subseteq \mathbb{P}^{1} \times \mathbb{P}^{1}$ be a set of points with $I_{\mathbb{X}} \subseteq\left(m_{1} \otimes m_{2}\right)^{\perp}$. Let $\mathbb{X}^{\prime}=\mathbb{X} \backslash\left\{x_{1}=0\right\}$ : we have $I_{\mathbb{X}^{\prime}}=I_{\mathbb{X}}:\left(x_{1}\right)$ and $x_{1}$ is not a zero-divisor in $S / I_{\mathbb{X}^{\prime}}$. This implies that, for $i \gg 1$ and for $j \geq 1$ :

$$
|\mathbb{X}| \geq\left|\mathbb{X}^{\prime}\right| \geq \operatorname{HF}_{S / I_{\mathbb{X}}^{\prime}}(i, j)=\sum_{k=1}^{i} \operatorname{HF}_{S / I_{\mathbb{X}^{\prime}}+\left(x_{1}\right)}(k, j) \text {. }
$$

Now, since $I_{\mathbb{X}} \subset\left(m_{1} \otimes m_{2}\right)^{\perp}$, we have $I_{\mathbb{X}^{\prime}} \subset\left(m_{1} \otimes m_{2}\right)^{\perp}:\left(x_{1}\right)$, so that

$$
\sum_{k \geq 0} \operatorname{HF}_{S / I_{\mathbb{X}^{\prime}}+\left(x_{1}\right)}(k, j) \geq \sum_{k \geq 0} \mathrm{HF}_{S /\left(\left(m_{1} \otimes m_{2}\right)^{\perp}:\left(x_{1}\right)+\left(x_{1}\right)\right)}(k, j) .
$$

Let $J=\left(m_{1} \otimes m_{2}\right)^{\perp}:\left(x_{1}\right)+\left(x_{1}\right)=\left(x_{1}, y_{1}^{b+1}, x_{2}^{a+1}, y_{2}^{b+1}\right)$. We have

$$
S / J \simeq \mathbb{k}\left[x_{1}, y_{1}\right] /\left(x_{1}, y_{1}^{b_{1}+1}\right) \otimes \mathbb{k}\left[x_{2}, y_{2}\right] /\left(x_{2}^{a_{2}+1}, y_{2}^{b_{2}+1}\right) .
$$

Therefore,

$$
\sum_{k \geq 0} \mathrm{HF}_{S / J}(k, j)=\sum_{k \geq 0} \operatorname{dim}\left(\mathbb{k}\left[x_{1}, y_{1}\right] /\left(x_{1}, y_{1}^{b_{1}+1}\right)\right)_{(k)} \cdot \operatorname{dim}\left(\mathbb{k}\left[x_{2}, y_{2}\right] /\left(x_{2}^{a_{2}+1}, y_{2}^{b_{2}+1}\right)\right)_{(j)} .
$$

Let $j=a_{2}$, so that the right hand side is $\left(b_{1}+1\right)\left(a_{2}+1\right)$. We conclude that

$$
\mathrm{R}_{\mathcal{C}_{a_{1}+b_{1}} \times \mathcal{C}_{a_{2}+b_{2}}}\left(m_{1} \otimes m_{2}\right) \geq|\mathbb{X}| \geq\left|\mathbb{X}^{\prime}\right| \geq\left(b_{1}+1\right)\left(a_{2}+1\right)
$$

Exchanging the roles of $m_{1}$ and $m_{2}$, we get $\mathrm{R}_{\mathcal{C}_{a_{1}+b_{1}} \times \mathcal{C}_{a_{2}+b_{2}}}\left(m_{1} \otimes m_{2}\right) \geq\left(b_{2}+1\right)\left(a_{1}+1\right)$.

The bound of Proposition 4.3 is far from being sharp: recall that $\mathrm{R}_{\mathbb{C}_{3} \times \mathbb{C}_{3}}\left(x^{2} y \otimes x^{2} y\right)=8[\mathrm{CF} 18$, BBCG19], while the lower bound from Proposition 4.3 is just 6 .

\section{Minimal DeCompositions of products and produCts of Minimal DeCOMpositions}

In this section, we focus on cases in which multiplicativity of rank holds. In particular, we ask whether minimal decompositions of a tensor product always arise as tensor products of minimal decompositions of the factors. 
We consider varieties $X_{1}, \ldots, X_{k}$, with $X_{i} \subset \mathbb{P} V_{i}$, for all $i=1, \ldots, k$. Given sets of points $S_{1}, \ldots, S_{k}$ with $S_{i} \subset \mathbb{P} V_{i}$, denote by $S_{1} \times \ldots \times S_{k}$ both the cartesian product in $\mathbb{P} V_{1} \times \ldots \mathbb{P} V_{k}$ and its image with respect to the Segre embedding $\mathbb{P} V_{1} \times \ldots \times \mathbb{P} V_{k} \rightarrow \mathbb{P}\left(V_{1} \otimes \cdots \otimes V_{k}\right)$, i.e., the set $\left\{z_{1} \otimes \ldots \otimes z_{k} \mid z_{i} \in S_{i}\right\}$.

First, we provide an immediate result on minimality of product decompositions.

Lemma 5.1. For $i=1, \ldots, k$, let $X_{i} \subset \mathbb{P} V_{i}$ be a variety, let $p_{i} \in X_{i}$ and let $S_{i} \subseteq X_{i}$ be a non redundant set of points spanning $p_{i}$, namely $p_{i} \in\left\langle S_{i}\right\rangle$ and no proper subset of $S_{i}$ spans $p_{i}$. Let $S=S_{1} \times \cdots \times S_{k}$. Then, there is no proper subset $T \subsetneq S \subset \mathbb{P}\left(V_{1} \otimes \cdots \otimes V_{k}\right)$ such that $p_{1} \otimes \cdots \otimes p_{k} \in\langle T\rangle$.

Proof. We proceed by induction on $k$. Let $k=2$. Write $S_{1}=\left\{a_{1}, \ldots, a_{s_{1}}\right\}$ and $S_{2}=\left\{b_{1}, \ldots, b_{s_{2}}\right\}$. Without loss of generality, we may assume $p_{1}=\sum_{i} a_{i}$ and $p_{2}=\sum_{i} b_{i}$. So $p_{1} \otimes p_{2}$ can be regarded as an element of the space of matrices $\left\langle S_{1}\right\rangle \otimes\left\langle S_{2}\right\rangle \subseteq V_{1} \otimes V_{2}$. In particular, the set $S_{1} \times S_{2}=$ $\left\{a_{i} \otimes b_{j}: i=1, \ldots, s_{1}, j=1, \ldots, s_{2}\right\}$ gives a basis for $\left\langle S_{1}\right\rangle \otimes\left\langle S_{2}\right\rangle$ and we can choose coordinates so that $a_{i} \otimes b_{j}$ is represented by the matrix having the $(i, j)$-th entry equal to 1 and zero elsewhere, and $p_{1} \otimes p_{2}$ is represented by the matrix having 1 in every entry. We conclude that no proper $T \subsetneq S_{1} \times S_{2}$ can span $p_{1} \otimes p_{2}$ because every element of $\langle T\rangle$ has a zero entry.

If $k \geq 2$, the statement follow by induction regarding $p_{1} \otimes \cdots \otimes p_{k}$ as $p_{1} \otimes\left(p_{2} \otimes \cdots \otimes p_{k}\right)$.

Notation 5.2. Given a Cartier divisor $D$ and a set of points $A$, we denote by $\operatorname{Res}_{D}(A)$ the residual set of points with respect to $A$, namely the set $A \backslash(A \cap D)$. In particular, if $\mathcal{I}_{A}$ and $\mathcal{I}_{D}$ are the ideal sheaves defining $A$ and $D$, respectively, then $\mathcal{I}_{\operatorname{Res}_{D}(A)}=\mathcal{I}_{A}: \mathcal{I}_{D}$.

In Theorem 5.4, we will use a slight variant of [BBCG19, Lemma 2.5]. Given a variety $X$, a very ample line bundle $\mathcal{L}$ on $X$, and a set of points $S \subseteq X$, we say that $S$ imposes independent conditions on the sections of $\mathcal{L}$ if $h^{1}\left(\mathcal{I}_{S} \otimes \mathcal{L}\right)=h^{1}(\mathcal{L})$ or equivalently if the restriction map $H^{0}(\mathcal{L}) \rightarrow H^{0}\left(\left.\mathcal{L}\right|_{S}\right)$ is surjective.

Lemma 5.3. Let $X$ be a variety and let $\mathcal{L}$ be a very ample line bundle on $X$. Let $V=H^{0}(\mathcal{L})^{*}$ and identify $X$ with its embedding in $\mathbb{P} V$. Let $p \in \mathbb{P} V$ and let $A, B \subset X$ be non redundant sets of points spanning $p$ in $\mathbb{P} V$. Assume $h^{1}\left(\mathcal{I}_{B} \otimes \mathcal{L}\right)=h^{1}(\mathcal{L})$. Let $D$ be an effective Cartier divisor on $X$ such that $\operatorname{Res}_{D}(A) \cap \operatorname{Res}_{D}(B)=\emptyset$. If $h^{1}\left(\mathcal{I}_{\operatorname{Res}_{D}(A \cup B)} \otimes \mathcal{L}(-D)\right)=0$ then $A \cup B \subseteq D$.

Proof. The proof is essentially the same as [BBCG19, Lemma 2.5] with the bundle $\mathcal{L}$ replacing the bundle $\mathcal{O}_{\mathbb{P}^{N}}(1)$. The condition $h^{1}\left(\mathcal{I}_{B}(1)\right)=0$ in [BBCG19, Lemma 2.5] is replaced by $h^{1}\left(\mathcal{I}_{B} \otimes \mathcal{L}\right)=$ $h^{1}(\mathcal{L})$ and the same argument provides the proof.

Recall the definition of identifiability: given $X \subseteq \mathbb{P}^{N}$ and $p \in \mathbb{P}^{N}$ with $\mathrm{R}_{X}(p)=r$, one says that $p$ is identifiable if there is a unique set of $r$ points of $X$ whose span contains $p$. We refer to [Chi19] for a basic introduction to identifiability problems.

Theorem 5.4. Let $X_{1}, \ldots, X_{k}$ be irreducible projective varieties. For every $i=1, \ldots, k$, let $\mathcal{L}_{i}$ be a very ample line bundle on $X_{i}$ and identify $X_{i}$ with the embedded subvariety in $V_{i}=H^{0}\left(\mathcal{L}_{i}\right)^{*}$ defined by the sections of $\mathcal{L}_{i}$. Let $\mathcal{M}_{i}$ and $\mathcal{N}_{i}$ be line bundles on $X_{i}$ with an isomorphism $\mathcal{M}_{i} \otimes \mathcal{N}_{i} \simeq \mathcal{L}_{i}$. Let $p_{i} \in \mathbb{P} V_{i}$ and $S_{i} \subseteq X_{i}$ be a set of points evincing $\mathrm{R}_{X_{i}}\left(p_{i}\right)$, for every $i=1, \ldots, k$. Then:

(i) If for every $i=1, \ldots, k, S_{i}$ imposes independent conditions on the sections of $\mathcal{N}_{i}, \mathcal{M}_{i}$ and $\mathcal{L}_{i}$ and in addition $h^{1}\left(M_{i}\right)=0$, then

$$
\mathrm{R}_{X_{1} \times \cdots \times X_{k}}\left(p_{1} \otimes \cdots \otimes p_{k}\right)=\prod_{i=1}^{k} \mathrm{R}_{X_{i}}\left(p_{i}\right) .
$$


(ii) If for every $i=1, \ldots, k, \mathcal{I}_{S_{i}} \otimes \mathcal{N}_{i}$ has no base points outside $S_{i}$, then $S_{1} \times \cdots \times S_{k}$ is the unique set of points evincing $\mathrm{R}_{X_{1} \times \cdots \times X_{k}}\left(p_{1} \otimes \cdots \otimes p_{k}\right)$. In particular, the $p_{i}$ 's are identifiable as well as $p_{1} \otimes \cdots \otimes p_{k}$.

Proof. Set $r_{i}=\mathrm{R}_{X_{i}}\left(p_{i}\right)$. Let $A \subseteq X_{1} \times \cdots \times X_{k}$ be a set of points enhancing the rank of $p_{1} \otimes \cdots \otimes p_{k}$. We show that $\operatorname{deg}(A) \geq \prod r_{i}$. Write $\mathcal{L}=\mathcal{L}_{1} \otimes \cdots \otimes \mathcal{L}_{k}$, regarded as a line bundle on $X_{1} \times \cdots \times X_{k}$ and similarly for $\mathcal{M}$ and $\mathcal{N}$.

Let $B=S_{1} \times \cdots \times S_{k}$. By Lemma 5.1, there is no proper subset of $B$ spanning $p_{1} \otimes \cdots \otimes p_{k}$. We will show $H^{0}\left(\mathcal{I}_{A} \otimes \mathcal{N}\right) \subseteq H^{0}\left(\mathcal{I}_{B} \otimes \mathcal{N}\right)$. If $H^{0}\left(\mathcal{I}_{A} \otimes \mathcal{N}\right)=0$, this statement is true. Otherwise, let $D \in H^{0}\left(\mathcal{I}_{A} \otimes \mathcal{N}\right)$ and identify $D$ with the divisor in $X_{1} \times \cdots \times X_{k}$ that it defines.

We verify the hypotheses of Lemma 5.3 to show $A \cup B \subseteq D$ :

- $B$ imposes independent conditions on $\mathcal{L}$ : this is straightforward from Künneth's formula as $H^{0}\left(\mathcal{L}_{1} \otimes \cdots \otimes \mathcal{L}_{k}\right)=\otimes H^{0}\left(\mathcal{L}_{i}\right)$ and similarly for the restrictions to $S$

- $\operatorname{Res}_{D}(A) \cap \operatorname{Res}_{D}(B)=\emptyset$, because $\operatorname{Res}_{D}(A)=\emptyset$ since $A \subseteq D$;

- $h^{1}\left(\mathcal{I}_{\operatorname{Res}_{D}(A \cup B)} \otimes \mathcal{L}(-D)\right)=0$ : notice $\mathcal{L}(-D)=\mathcal{M}$ because $\mathcal{L}=\mathcal{M} \otimes \mathcal{N}$ [Har77, Prop. II.6.13]; moreover $\operatorname{Res}_{D}(A \cup B)=\operatorname{Res}_{D}(B) \subseteq B$ and $B$ imposes independent conditions on $\mathcal{M}$, which guarantees $h^{1}\left(\mathcal{I}_{\operatorname{Res}_{D}(A \cup B)} \otimes \mathcal{L}(-D)\right) \leq h^{1}\left(\mathcal{I}_{B} \otimes \mathcal{M}\right)=h^{1}(\mathcal{M})$, where the right-hand-side is equal to 0 , again by Künneth's formula.

Applying Lemma 5.3, deduce $A \cup B \subseteq D$ and so $B \subseteq D$, and we deduce $H^{0}\left(\mathcal{I}_{A} \otimes \mathcal{N}\right) \subseteq H^{0}\left(\mathcal{I}_{B} \otimes \mathcal{N}\right)$. Again, $B$ imposes independent conditions on $\mathcal{N}$, namely the restriction map $H^{0}(\mathcal{N}) \rightarrow H^{0}\left(\left.\mathcal{N}\right|_{B}\right)$ is surjective and one has the exact sequence

$$
0 \rightarrow H^{0}\left(\mathcal{I}_{B} \otimes \mathcal{N}\right) \rightarrow H^{0}(\mathcal{N}) \rightarrow H^{0}\left(\left.\mathcal{N}\right|_{B}\right) \rightarrow 0
$$

This provides $h^{0}\left(\mathcal{I}_{B} \otimes \mathcal{N}\right)=h^{0}(\mathcal{N})-\operatorname{deg}(B)=h^{0}(\mathcal{N})-\prod_{i} r_{i}$. On the other hand $h^{0}\left(\mathcal{I}_{B} \otimes \mathcal{N}\right) \geq$ $h^{0}\left(\mathcal{I}_{A} \otimes \mathcal{N}\right) \geq h^{0}(\mathcal{N})-\operatorname{deg}(A)$. Hence, $\operatorname{deg}(A) \geq \prod_{i} r_{i}$ and part (i) of the statement holds.

It remains to show that if the hypothesis of (ii) is satisfied, then $A=B$. From the first part of the proof we have $\operatorname{deg}(A)=\operatorname{deg}(B)$. Then, $H^{0}\left(\mathcal{I}_{A} \otimes \mathcal{N}\right)=H^{0}\left(\mathcal{I}_{B} \otimes \mathcal{N}\right)$. Indeed: $h^{0}\left(\mathcal{I}_{B} \otimes \mathcal{N}\right)=h^{0}(\mathcal{N})-$ $\operatorname{deg}(B)$, because $B$ imposes independent conditions on $\mathcal{N}$ and, on the other hand, $h^{0}\left(\mathcal{I}_{A} \otimes \mathcal{N}\right)=$ $h^{0}(\mathcal{N})-\operatorname{deg}(A)+h^{1}\left(\mathcal{I}_{A} \otimes \mathcal{N}\right)-h^{1}(\mathcal{N}) ;$ notice $h^{1}\left(\mathcal{I}_{A} \otimes \mathcal{N}\right)-h^{1}(\mathcal{N}) \geq 0$ because $H^{1}\left(\mathcal{I}_{A} \otimes \mathcal{N}\right) \rightarrow H^{1}(\mathcal{N})$ is surjective. This shows $h^{0}\left(\mathcal{I}_{B} \otimes \mathcal{N}\right)=h^{0}\left(\mathcal{I}_{A} \otimes \mathcal{N}\right)$ and, since from the first part of the proof $H^{0}\left(\mathcal{I}_{A} \otimes \mathcal{N}\right) \subseteq H^{0}\left(\mathcal{I}_{B} \otimes \mathcal{N}\right)$, the equality follows.

Now, by assumption $\mathcal{I}_{S_{i}} \otimes \mathcal{N}_{i}$ has no base points out of $S_{i}$, and therefore $\mathcal{I}_{B} \otimes \mathcal{N}$ has no base points outside of $B$ : this shows $A \subseteq B$ and since they have the same degree equality holds.

Theorem 5.4 can be applied to Veronese varieties as follows.

Corollary 5.5. Let $i=1, \ldots, k$. For every $i$, let $d_{i} \geq 1, n_{i} \geq 1$ and let $f_{i} \in \mathbb{P} \operatorname{Sym}^{d_{i}} \mathbb{C}^{n_{i}+1}$ be elements with $\mathrm{R}_{\nu_{d_{i}}\left(\mathbb{P}^{n_{i}}\right)}\left(f_{i}\right)=r_{i} \leq\left\lceil d_{i} / 2\right\rceil$. Let $S_{i} \subseteq \mathbb{P}^{n_{i}}$ be a set of $r_{i}$ points such that $p_{i} \in\left\langle\nu_{d_{i}}\left(S_{i}\right)\right\rangle$. Then $\mathrm{R}_{\nu_{d_{1}}, \ldots, d_{k}}\left(\mathbb{P}^{n} n_{\left.1 \times \cdots \times \mathbb{P}^{n} k\right)}\left(f_{1} \otimes \cdots \otimes f_{k}\right)=r_{1} \cdots r_{k}\right.$ and $S_{1} \times \cdots \times S_{k}$ is the unique set of points in $\mathbb{P}^{n_{1}} \times \cdots \times \mathbb{P}^{n_{k}}$ such that $p_{1} \otimes \cdots \otimes p_{k} \in\left\langle\nu_{d_{1}, \ldots, d_{k}}\left(S_{1} \times \cdots \times S_{k}\right)\right\rangle$.

We point out that in general, already for points of rank two, there are decompositions of the product not arising from decompositions of the single factors.

Example 5.6. Let $X_{1} \subseteq \mathbb{P}^{N_{1}}$ be a variety; suppose there is a 2-dimensional linear space $\mathbb{P} A$ such that $\mathbb{P} A \cap X_{1}$ contains at least four points of intersection $a_{1}, a_{2}, a_{3}, a_{4} \in X_{1}$. Let $p_{1} \in\left\langle a_{1}, a_{2}\right\rangle$ be a point such that $\underline{\mathrm{R}}_{X_{1}}\left(p_{1}\right)=2$. 
Let $X_{2} \subseteq \mathbb{P}^{N_{2}}$ be a variety, let $b_{1}, b_{2} \in X_{2}$ be two points and let $p_{2} \in\left\langle b_{1}, b_{2}\right\rangle$ be a point such that $\mathrm{R}_{X_{2}}\left(p_{2}\right)=2$. Write $p_{1}=a_{1}+a_{2}$ and $p_{2}=b_{1}+b_{2}$.

Suppose that the condition described in Theorem 2.6 does not hold, so that $\mathrm{R}_{X_{1} \times X_{2}}\left(p_{1} \otimes p_{2}\right)=4$. Then $\left\{a_{1}, a_{2}\right\} \times\left\{b_{1}, b_{2}\right\}=\left\{a_{1} \otimes b_{1}, a_{2} \otimes b_{1}, a_{1} \otimes b_{2}, a_{2} \otimes b_{2}\right\}$ is a minimal set of four points $X_{1} \times X_{2}$ spanning $p_{1} \otimes p_{2}$.

We determine a second set of four points as follows. After a suitable choice of coordinates, write $a_{4}=a_{1}+a_{2}-a_{3}$. Define

$$
\begin{aligned}
& a_{1}^{\prime}=a_{1}, \quad a_{2}^{\prime}=a_{2}, \quad a_{3}^{\prime}=a_{3}, \quad a_{4}^{\prime}=a_{4}, \\
& b_{1}^{\prime}=b_{1}, \quad b_{2}^{\prime}=b_{1}, \quad b_{3}^{\prime}=b_{2}, \quad b_{4}^{\prime}=b_{2},
\end{aligned}
$$

Then

$$
\begin{aligned}
\sum_{i} a_{i}^{\prime} \otimes b_{i}^{\prime} & =a_{1} \otimes b_{1}+a_{2} \otimes b_{1}+a_{3} \otimes b_{2}+\left(a_{1}+a_{2}-a_{3}\right) \otimes b_{2} \\
& =\left(a_{1}+a_{2}\right) \otimes\left(b_{1}+b_{2}\right)=p_{1} \otimes p_{2} .
\end{aligned}
$$

This shows that $S=\left\{a_{1}^{\prime} \otimes b_{1}^{\prime}, \ldots, a_{4}^{\prime} \otimes b_{4}^{\prime}\right\}$ is a set of four points of $X_{1} \times X_{2}$ spanning $p_{1} \otimes p_{2}$. The set $S$ is not of the form $S_{1} \times S_{2}$ for some $S_{1} \subseteq X_{1}, S_{2} \subseteq X_{2}$.

\section{REFERENCES}

[Ådl87] B. Ådlandsvik, Joins and higher secant varieties, Mathematica Scandinavica 61 (1987), $213-222$.

[AR08] E. S. Allman and J. A. Rhodes, Phylogenetic ideals and varieties for the general Markov model, Adv. Appl. Math. 40 (2008), no. 2, 127-148.

[Bar06] P. Le Barz, Sur les espaces multisécants aux courbes algébriques, Manuscripta Math. 119(4) (2006), 433-452.

[BB14] W. Buczyńska and J. Buczyński, Secant varieties to high degree Veronese reembeddings, catalecticant matrices and smoothable Gorenstein schemes, J. Algebraic Geom. 23 (2014), no. 1, 63-90.

[BBC18] E. Ballico, A. Bernardi, and L. Chiantini, On the dimension of contact loci and the identifiability of tensors, Arkiv för Matematik 56 (2018), no. 2, 265-283.

[BBCG19] E. Ballico, A. Bernardi, M. Christandl, and F. Gesmundo, On the partially symmetric rank of tensor products of $W$-states and other symmetric tensors, Atti Accad. Naz. Lincei Rend. Lincei Mat. Appl. 30 (2019), 93-124.

[BBCM11] A. Bernardi, J. Brachat, P. Comon, and B. Mourrain, Multihomogeneous polynomial decomposition using moment matrices, Proc. 36th Int. Symp. Symb. Alg. Comp. - ISSAC'11, ACM Press, 2011.

[BBG19] E. Ballico, A. Bernardi, and F. Gesmundo, A note on the cactus rank for Segre-Veronese varieties, J. Algebra 526 (2019), 6 - 11.

$\left[\mathrm{BCC}^{+} 18\right]$ A. Bernardi, E. Carlini, M. V. Catalisano, A. Gimigliano, and A. Oneto, The Hitchhiker guide to: secant varieties and tensor decomposition, Mathematics 6 (2018), no. 12, 314.

[Bea96] A. Beauville, Complex algebraic surfaces, Student Texts, vol. 34, London Mathematical Society, London, 1996.

[Ber98] M.-A. Bertin, On the singularities of the trisecant surface to a space curve, Le Matematiche (Catania) 53 (1998), no. 3, 15-22.

[Ber08] A. Bernardi, Ideals of varieties parameterized by certain symmetric tensors, J. Pure Applied Alg. 212 (2008), no. 6, 1542-1559.

[BGI11] A. Bernardi, A. Gimigliano, and M. Idà, Computing symmetric rank for symmetric tensors, J. Symb. Comp. 46 (2011), 34-53.

[BGL13] J. Buczyński, A. Ginensky, and J. M. Landsberg, Determinantal equations for secant varieties and the Eisenbud-Koh-Stillman conjecture, Journal of the London Mathematical Society 88 (2013), no. 1, 1-24.

[BPR20] J. Buczyński, E. Postinghel, and F. Rupniewski, On Strassen's rank additivity for small three-way tensors, SIAM Journal on Matrix Analysis and Applications 41 (2020), no. 1, 106-133.

[BR13] A. Bernardi and K. Ranestad, On the cactus rank of cubic forms, J. Symb. Comp. 50 (2013), $291-297$.

[CC02] L. Chiantini and C. Ciliberto, Weakly defective varieties, Trans. Amer. Math. Soc. 454(1) (2002), 151178.

[CC06] - On the concept of k-secant order of a variety, J. London Math. Soc. 72(2) (2006), 151-178.

[CCC15] E. Carlini, M. V. Catalisano, and L. Chiantini, Progress on the symmetric Strassen conjecture, Journal of Pure and Applied Algebra 219 (2015), no. 8, 3149-3157. 
$\left[\mathrm{CCC}^{+} 18\right]$ E. Carlini, M. V. Catalisano, L. Chiantini, A. V. Geramita, and Y. Woo, Symmetric tensors: rank, Strassen's conjecture and e-computability, Ann. Sc. Norm. Super. Pisa Cl. Sci. XVIII (2018), no. 1, 363-390.

[CCG12] E. Carlini, M. V. Catalisano, and A. V. Geramita, The solution to the Waring problem for monomials and the sum of coprime monomials, J. Algebra 370 (2012), 5-14.

[CCO17] E. Carlini, M. V. Catalisano, and A. Oneto, Waring loci and the Strassen conjecture, Adv. Math. 314 (2017), 630-662.

[CF18] L. Chen and S. Friedland, The tensor rank of tensor product of two three-qubit $W$ states is eight, Lin. Alg. Appl. 543 (2018), 1-16.

[CGJ19] M. Christandl, F. Gesmundo, and A. K. Jensen, Border rank is not multiplicative under the tensor product, SIAM J. Appl. Alg. Geom. 3 (2019), 231-255.

[Chi19] L. Chiantini, Hilbert Functions and Tensor Analysis, Ballico E., Bernardi A., Carusotto I., Mazzucchi S., Moretti V. (eds) Quantum Physics and Geometry, vol. 25, pp. 125-151, Springer, Cham, 2019.

[CJ01] M. L. Catalano-Johnson, The homogeneous ideals of higher secant varieties, J. Pure Applied Alg. 158 (2001), no. 2-3, 123-129.

[CJZ18] M. Christandl, A. K. Jensen, and J. Zuiddam, Tensor rank is not multiplicative under the tensor product, Lin. Alg. Appl. 543 (2018), 125-139.

[Cle61] A. Clebsch, Uber Curven fierter Ordnung, J. Reine Angew. Math. 59 (1861), 125-145.

[CS11] G. Comas and M. Seiguer, On the rank of a binary form, Found. of Comp. Math. 11 (2011), no. 1, 65-78.

[DVC00] W. Dür, G. Vidal, and J. I. Cirac, Three qubits can be entangled in two inequivalent ways, Phys. Rev. A 62 (2000), no. 6, 062314, 12.

[Eis95] D. Eisenbud, Commutative Algebra: with a view toward algebraic geometry, Graduate Texts in Mathematics, vol. 150, Springer-Verlag, New York, 1995.

[Gał16] M. Gałązka, Multigraded apolarity, arXiv:1601.06211 (2016).

[Ger96] A. V. Geramita, Inverse systems of fat points: Waring's problem, secant varieties of Veronese varieties and parameter spaces for Gorenstein ideals, The curves seminar at Queen's, vol. 10, 1996, pp. 2-114.

[GRV16] M. Gallet, K. Ranestad, and N. Villamizar, Varieties of apolar subschemes of toric surfaces, Arkiv för Matematik 56 (2016), no. 1, 73-99.

[Har77] R. Hartshorne, Algebraic Geometry, Graduate Texts in Mathematics, Springer-Verlag, Berlin-HeidelbergNew York, 1977.

[Har92] J. Harris, Algebraic geometry. A first course, Graduate Texts in Mathematics, vol. 133, Springer-Verlag, New York, 1992.

[IK99] A. Iarrobino and V. Kanev, Power sums, Gorenstein algebras, and determinantal loci, Springer Science \& Business Media, 1999.

[Kaj86] H. Kaji, On the tangentially degenerate curves, J. London Math. Soc. (2) 33(3) (1986), 430-440.

[KM02] I. A. Kogan and M. M. Maza, Computation of canonical forms for ternary cubics, Proc. 2002 Int. Symp. Symb. Alg. Comp., ACM, 2002, pp. 151-160.

[Lan12] J. M. Landsberg, Tensors: Geometry and Applications, vol. 128, American Mathematical Soc., 2012.

[LM17] J. M. M Landsberg and M. Michałek, Abelian tensors, Journal de Mathématiques Pures et Appliquées 108 (2017), no. 3, 333-371.

[LO13] J. M. Landsberg and G. Ottaviani, Equations for secant varieties of Veronese and other varieties, Ann. Mat. Pura Appl. (4) 192 (2013), no. 4, 569-606.

[LT10] J. M. Landsberg and Z. Teitler, On the ranks and border ranks of symmetric tensors, Found. of Comp. Math. 10 (2010), no. 3, 339-366.

[Mil08] J. S. Milne, Abelian Varieties, 2008, Course Notes available at http://www.jmilne.org/math/CourseNotes/AV.pdf.

[Pal03] F. Palatini, Sulla rappresentazione delle forme ed in particolare della cubica quinaria con la somme di potenze di forme lineari, Atti R. Accad. Sc. Torino 38 (1902-1903), 43-50.

[Pal06] Sulle superficie algebriche $i$ cui $S_{h}(h+1)$-seganti non riempiono lo spazio ambiente, Atti Accad. Torino 41 (1906), 634-640.

[RS11] K. Ranestad and F.-O. Schreyer, On the rank of a symmetric form, J. Algebra 346 (2011), no. 1, 340-342.

[Rus16] F. Russo, On the Geometry of Some Special Projective Varieties, Lecture Notes of the Unione Matematica Italiana, vol. 18, Springer, 2016.

[Sch81] A. Schönhage, Partial and total matrix multiplication, SIAM Journal on Computing 10 (1981), no. 3, 434-455.

[Shi19] Y. Shitov, Counterexamples to Strassen's direct sum conjecture, Acta Mathematica 222 (2019), no. 2, $363-379$.

[Str69] V. Strassen, Gaussian elimination is not optimal, Numerische mathematik 13 (1969), no. 4, 354-356.

[Str73] _ _ Vermeidung von Divisionen, J. Reine Angew. Math. 264 (1973), 184-202. 
[Str87] _ Relative bilinear complexity and matrix multiplication, J. Reine Angew. Math. $375 / 376$ (1987), 406-443.

[SVT06] J. Sidman and A. Van Tuyl, Multigraded regularity: syzygies and fat points, Contrib. to Algebra and Geometry 47 (2006), no. 1, 1-22.

[Syl51] J. J. Sylvester, LX. On a remarkable discovery in the theory of canonical forms and of hyperdeterminants, The London, Edinburgh, and Dublin Philosophical Magazine and Journal of Science 2 (1851), no. 12, 391-410.

[Syl52] _ On the principles of the calculus of forms, Cambridge and Dublin Math. J. (1852), 52-97.

[Tei15] Z. Teitler, Sufficient conditions for Strassen's additivity conjecture, Illinois J. Math. 59 (2015), no. 4, 1071-1085.

(E. Ballico, A. Bernardi, A. Oneto) Dipartimento di Matematica, Università di Trento, 38123 Povo (TN), ITALY

(F. Gesmundo) QMATH, Dept. Math. Sciences, U. Copenhagen, Universitetsparken 5, 2100 Copenhagen O., Denmark

(E. Ventura) Universität Bern, Mathematisches Institut, Sidlerstrasse 5, 3012 Bern, Switzerland

E-mail address, Ballico: edoardo.ballico@unitn.it

E-mail address, Bernardi: alessandra.bernardi@unitn.it

E-mail address, Gesmundo: fulges@math.ku.dk

E-mail address, Oneto: alessandro.oneto@unitn.it

E-mail address, Ventura: emanueleventura.sw@gmail.com, emanuele.ventura@math.unibe.ch 